\title{
Catalytic and highly enantioselective Friedel-Crafts type reactions of heteroaromatic compounds with trifluoropyruvate and glyoxylate by a dicationic palladium complex
}

\author{
Kohsuke Aikawa, Yuya Asai, Yūta Hioki, Koichi Mikami* \\ Department of Applied Chemistry, Graduate School of Science and Engineering, Tokyo Institute of Technology, O-okayama, Meguro-ku, Tokyo 152-8552 (Japan)
}

\section{ARTICLE INFO}

Article history:

Received

Received in revised form

Accepted

Available online

\section{ABSTRACT}

The highly enantioselective Friedel-Crafts alkylation of furan and thiophene derivatives with trifluoropyruvate, which have never provided the high level of asymmetric induction and yield until now, was achieved by using dicationic palladium complexes as Lewis acid catalysts. Moreover, glyoxylate instead of trifluoropyruvate as an electrophile led to complete change of regioselectivity with 2-trimethylsilylated furan, thiophene, and pyrrole derivatives to give the corresponding heteroarylated products in high yields and enantioselectivities. The respective products with trifluoropyruvate and glyoxylate could also be obtained via sequential catalytic reaction; intramolecular cyclization of alkynyldiols using cationic Au catalyst followed by FRIEDEL-CRAFTS type reactions using dicationic Pd catalyst.

2009 Elsevier Ltd. All rights reserved.

\section{Introduction}

Organofluorine compounds, which are extremely rare in Nature, have received considerable attention in the fields of pharmaceuticals, agrochemicals, and organic materials due to their unique chemical, biological, and physical properties. ${ }^{1}$ In modern medicinal chemistry, trifluoromethyl-substituted compounds are widely employed, because trifluoromethyl $\left(\mathrm{CF}_{3}\right)$ groups can bring higher metabolic stability, increased lipophilicity, and stronger dipole moments to druglike compounds. ${ }^{1}$ Therefore, a variety of synthetic methods to introduce the $\mathrm{CF}_{3}$ group into organic compounds have been reported to readily produce the corresponding compounds, even in optically active forms. Optically active $\alpha$-trifluoromethylsubstituted tertiary alcohols prepared via catalytic asymmetric synthesis have attracted widespread interest due to their unique biological activities, as typically shown in the anti-HIV agent (Efavirenz) $^{2}$ and nonsteroidal glucocorticoid receptor (GR) agonists. $^{3}$

The Friedel-Crafts alkylation is one of the most fundamental carbon-carbon bond forming reactions in modern organic synthesis. $^{4}$ Chiral transition-metal catalysts as well as organocatalysts have been developed for the Friedel-Crafts alkylations of (hetero)aromatic compounds with carbonyl and imine compounds. ${ }^{4}$ The Friedel-Crafts alkylations by treatment of trifluoropyruvate as a carbonyl compound, which is a versatile and commercially available reagent for the $\mathrm{CF}_{3}$ building block method, can lead to optically active $\alpha$-trifluoromethyl-substituted tertiary alcohols in high yields and enantioselectivities. ${ }^{5-8}$ However, the high level of asymmetric induction is limited to indole, pyrrole, and activated aromatic derivatives as nucleophiles with higher reactivity, ${ }^{5}$ as compared with furan and thiophene derivatives. Herein, we report the highly enantioselective Friedel-Crafts alkylations of furan and thiophene derivatives with trifluoropyruvate with high levels of asymmetric induction and yield using dicationic palladium complexes as Lewis acid catalysts (Scheme 1-1). We also report that the combination of 2-trimethylsilylated furan, thiophene, and pyrrole derivatives and glyoxylate leads to a complete change of regioselectivity to afford the heteroarylated products at the 2-position along with high asymmetric induction (Schemes 1-2).

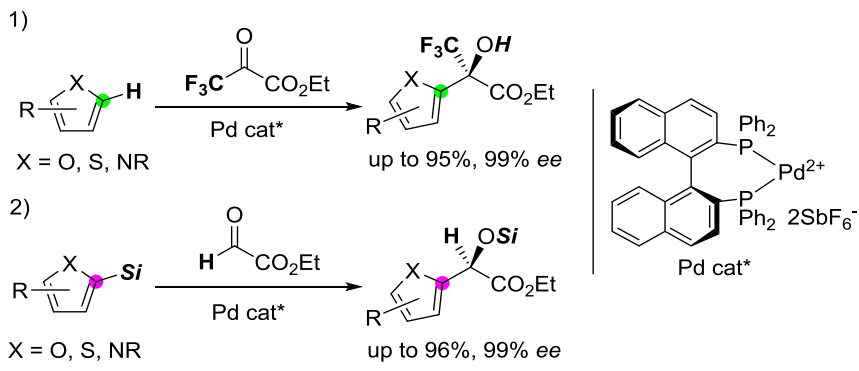

Scheme 1. Regio- and enantioselective Friedel-Crafts type reactions with dicationic palladium catalyst

\section{Results and discussion}

Initially, the Friedel-Crafts type reaction of 2-trimethylsilyl furan $\mathbf{4 a}$ as a nucleophile was examined in the presence of 
dicationic Pd-catalyst 1a $(5 \mathrm{~mol} \%)$ prepared in situ from the corresponding dichloride complex and two equivalents of $\mathrm{AgSbF}_{6}{ }^{10}$ (Scheme 2). As shown in the literature, ${ }^{9}$ the reactions with ethyl glyoxylate $\mathbf{3}$ proceeded regioselectively at the 2position even at $-40{ }^{\circ} \mathrm{C}$ to give product $\mathbf{6 a}$ in excellent yield and enantioselectivity after the desilylation of siloxy product $\mathbf{6 a}$ ' (Scheme 2, Eq. 2). In sharp contrast to ethyl glyoxylate 3, it was found that the reaction with ethyl trifluoropyruvate 2 provided the Friedel-Crafts product 5a at the 5-position with high levels of enantioselectivity but moderate yield, although 6a and 6a' were not observed at all (Scheme 2, Eq. 1). It is supposed that the complete change of regioselectivity originates from the steric repulsion between the trimethylsilyl and trifluoromethyl groups. The absolute configuration at the created carbon center of $\mathbf{5 a}$ and 6a was determined to be the same $(R)$ by comparison with the specific rotation of the reported data. ${ }^{5 \mathrm{~b}, 9}$
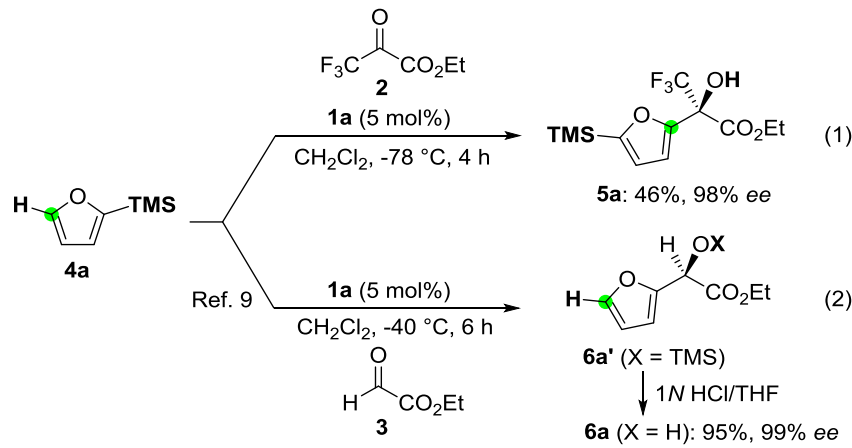

Scheme 2. Complete change of regioselectivity in reaction with 2 trimethylsilyl furan and carbonyl compounds

Encouraged by the excellent enantioselectivity $(98 \%$ ee $)$ obtained even with simple mono-substituted furan, we attempted to optimize the Friedel-Crafts alkylation (Table 1). (S)-BINAP and $(S)$-SEGPHOS exhibited excellent enantioselectivities, while the yields were moderate (entries 1 and 2). The more sterically demanding ligand, (S)-DTBM-SEGPHOS led to an almost racemic product (entry 3$)$. ( $R, R)$-QuinoxP $*^{11}$ also gave high levels of enantioselectivity (entry 4$)$. With the aim of enhancing the yield, we investigated the effect of the co-solvent. The use of toluene as a co-solvent did not increase the yield (entries 5-6). The combination of $\mathrm{CH}_{2} \mathrm{Cl}_{2}$ and the coordinating solvent $\mathrm{Et}_{2} \mathrm{O}$, which generally lowers the reactivity of Lewis acid catalysts, catalyzed the reaction to provide product 5a in high yields, while maintaining excellent enantioselectivities (entries 7-8). Additionally, the yield and enantioselectivity could be maintained even with a lower catalyst loading of 1 and $2 \mathrm{~mol} \%$ (90-93\% yields, 99\% ee) (entries 9-10). The use of THF or $\mathrm{CH}_{3} \mathrm{CN}$ provided no products (entries 11-12).

To extend the scope of the substrates, the Friedel-Crafts alkylation was further examined under the optimized reaction conditions in hand (Table 2). Non-substituted furan $\mathbf{4 b}$ exhibited excellent results $(95 \%, 99 \%$ ee), although it was hard to attain both high yield and enantioselectivity as in the previous reports. $^{5 \mathrm{a}, \mathrm{b}}$ Furans $\mathbf{4 c - e}$ bearing aliphatic and aromatic substituents at the 5-position also gave the good results, but $\mathbf{4 c}$ decreased both the yield and enantioselectivity. Furan $\mathbf{4 f}$ with a phenyl group at the 2,4-positions could be employed regardless of steric hindrance $(82 \%, 99 \% e e)$. The reaction of furan $\mathbf{4 g}$ with an electron-withdrawing ester group did not proceed even under the reflux conditions. Non- or methyl-substituted thiophenes $\mathbf{4 h}-\mathbf{i}$ were amenable to the highly enantioselective Friedel-Crafts reaction. The tert-butoxycarbonyl (Boc) group of pyrrole $\mathbf{4 j}$ had a deteriorating effect on the regioselectivity and gave a mixture of a 3:2 ratio in the 2- and 3-positions. Treatment of $N$-unprotected pyrrole $4 \mathbf{k}$ led to a single product at the 2-position but the enantioselectivity was low $(89 \%, 37 \% e e)$.

Table 1

Catalytic asymmetric Friedel-Crafts alkylation of 2-trimethylsilyl furan with trifluoropyruvate ${ }^{\mathrm{a}}$

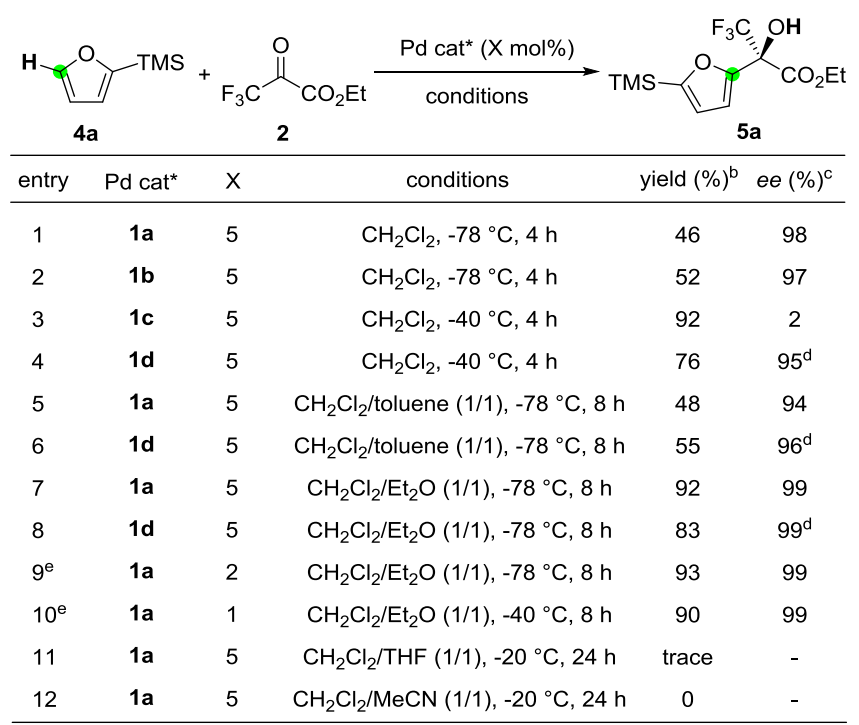

$\mathrm{a}_{3}$ equivalents of $\mathbf{2}$ were used. ${ }^{\mathrm{b}}$ Isolated yield. ${ }^{\mathrm{c}}$ Enantiopurity was determined by chiral HPLC anlysis. ${ }^{d}$ Opposite enantiomer $(S)$ was obtained. ${ }^{e_{1}} .2$ equivalents of 2 were used.

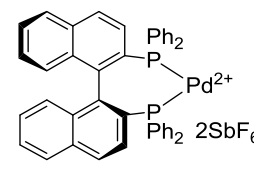

(S)-BINAP-Pd: $\mathbf{1 a}$

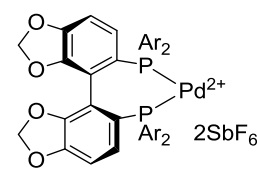

(S)-SEGPHOS-Pd $(\mathrm{Ar}=\mathrm{Ph})$ : 1b (S)-DTBM-SEGPHOS-Pd: 1c $\left(\mathrm{Ar}=3,5-{ }^{\mathrm{t}} \mathrm{Bu}_{2}-4-\mathrm{OMe}-\mathrm{C}_{6} \mathrm{H}_{2}\right)$

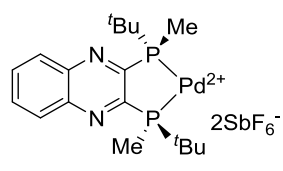

$(R, R)-Q u i n o x P^{*}:$ 1d
Table 2.

Scope of substrates in the catalytic enantioselective Friedel-Crafts alkylation

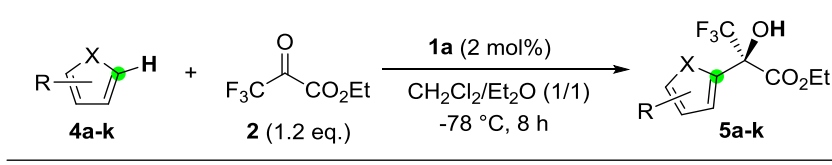

$$
\text { 5h: } 89 \%, 94 \% e e^{\mathrm{a}}
$$

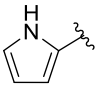

aConditions: $0{ }^{\circ} \mathrm{C}, 24 \mathrm{~h} .{ }^{\mathrm{b}}$ Conditions: $-40{ }^{\circ} \mathrm{C}, 24 \mathrm{~h} .{ }^{\mathrm{c}}$ Conditions: $-78^{\circ} \mathrm{C}, 24 \mathrm{~h}$

In sharp contrast to a heteroaromatic system, the combination of vinylsilane $\mathbf{7}$ as a nucleophile and ethyl trifluoropyruvate $\mathbf{2}$ afforded the alkenylated product $\mathbf{8}$ in $92 \%$ yield and with $98 \%$ ee after desilylation, due to the smaller steric repulsion between 7 and the trifluoromethyl group compared to $\mathbf{4 a}$ (Scheme 3, Eq. 1). Dienylsilane $\mathbf{9}$ also gave the dienylated product $\mathbf{1 0}$ in $82 \%$ and with $99 \%$ ee (Scheme 3, Eq. 2). 


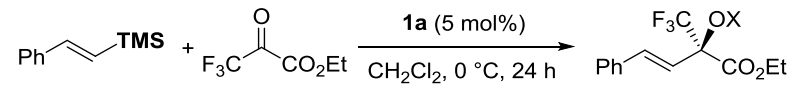

$$
\begin{aligned}
& 7 \quad 26 N \mathrm{NCI} / \mathrm{THF}^{-8}(\mathrm{X}=\mathrm{TMS})
\end{aligned}
$$

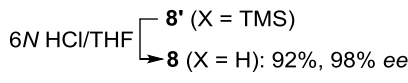

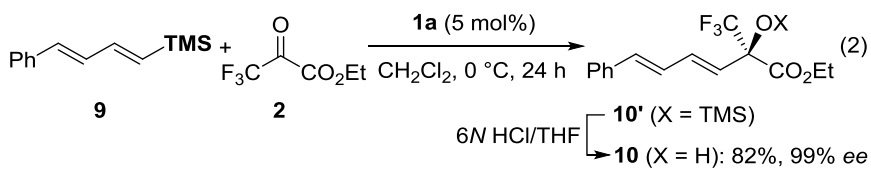

Scheme 3. Catalytic asymmetric alkenylation and dienylation

Table 3.

Catalytic asymmetric Friedel-Crafts type reaction of 2-trimethylsilylated furan, thiophene, and pyrrole derivatives with ethyl glyoxylate

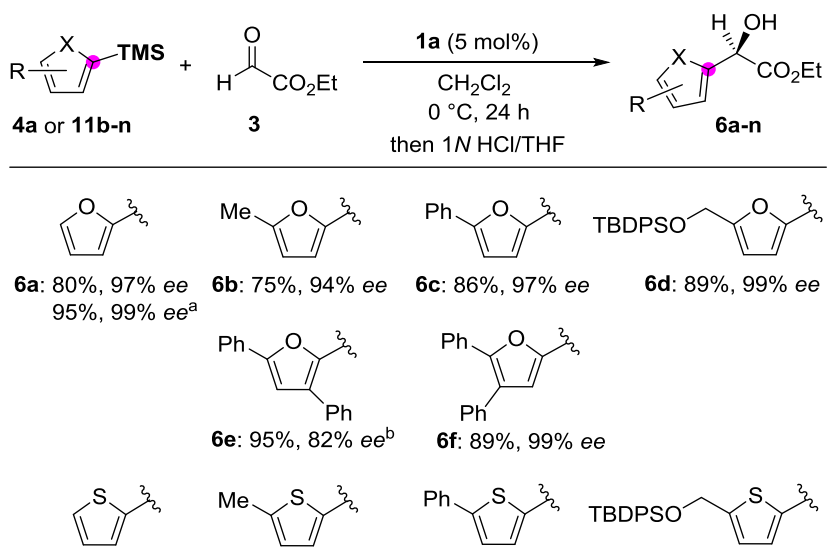

6g: $85 \%, 98 \% e e^{c} \quad$ 6h: $92 \%, 99 \% e e^{c} \quad 6 \mathbf{i}: 93 \%, 99 \% e e^{c} \quad$ 6j: $87 \%, 99 \% e e^{c}$

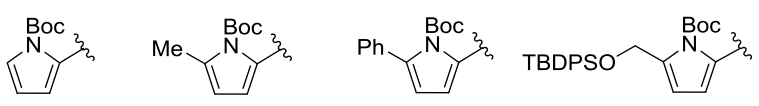

6k: $92 \%, 99 \%$ ee $e^{d}$ 6I: $96 \%, 98 \% e e^{e} \quad 6 \mathbf{m}: 91 \%, 96 \% e e^{e} \quad 6$ n: $85 \%, 99 \% e e^{e}$

${ }^{\mathrm{a} C}$ Conditions: $-40{ }^{\circ} \mathrm{C}, 6 \mathrm{~h} .{ }^{\mathrm{b}}$ Conditions: $-40{ }^{\circ} \mathrm{C}, 12 \mathrm{~h} .{ }^{\mathrm{c}}$ Conditions: $0{ }^{\circ} \mathrm{C}, 12 \mathrm{~h}$. ${ }^{\mathrm{d}}$ Conditions: $-78{ }^{\circ} \mathrm{C}, 5 \mathrm{~h}$. ${ }^{\mathrm{e}}$ Conditions: $-40^{\circ} \mathrm{C}, 24 \mathrm{~h}$.

We also investigated the scope of the substrates in catalytic asymmetric Friedel-Crafts type reactions of 2-trimethylsilylated heteroaromatic compounds with ethyl glyoxylate 3 (Table 3 ). All substrates gave products $\mathbf{6}$ bonded onto the trimethylsilylated carbon with high enantioselectivity. Not only mono-substituted furan $4 \mathbf{a}^{12}$ but also 2,5-disubstituted $\mathbf{1 1 b}$-d provided with high to excellent enantioselectivities (94-99\% ee). The reaction of 2,3,5trisubstituted furans 11e provided the product in high yield, but the enantioselectivity decreased with a deleterious effect on the steric hindrance $(95 \%, 82 \%$ ee $)$. 2,4,5-Trisubstituted furans $\mathbf{1 1 f}$ facilitated the reactions to give excellent results $(89 \%, 99 \%$ ee $)$. Additionally, the reactions with mono- and disubstituted thiophenes 11g-j also gave high yields and enantioselectivites (85-93\%, 98-99\% ee). The combination of Boc-pyrroles 11k-n and 3 led to complete regioselectivity at the 2-position and high enantioselectivities $(96-99 \% e e$ ), while the regioisomers at the 2and 3-positions were obtained in the Friedel-Crafts alkylation with Boc-pyrrole $\mathbf{4 j}$ and $\mathbf{2}$.

With these positive results for a wide range of 5-membered heteroaromatic nucleophiles, we utilized the heteroarylated products as chiral building blocks for the enantioselective synthesis of pyranone ${ }^{13}$ (Scheme 4). Reduction ${ }^{14}$ with 6a followed by pivaloyl protection provided the corresponding alcohol $\mathbf{1 3}$ in good yield. Reaction of NBS with $\mathbf{1 3}$ in the presence of $\mathrm{NaOAc}$ and $\mathrm{NaHCO}_{3}$ led to pyranone $\mathbf{1 4}$ via an
Achmatowicz rearrangement. ${ }^{15}$ Finally, Bz-protection of a hemiacetal proceeded to give the protected pyranone $\mathbf{1 5}^{\mathbf{1 3 b}}$ in a 4:1 ratio of trans and cis-stereoisomer in $86 \%$ yield for 2 steps.

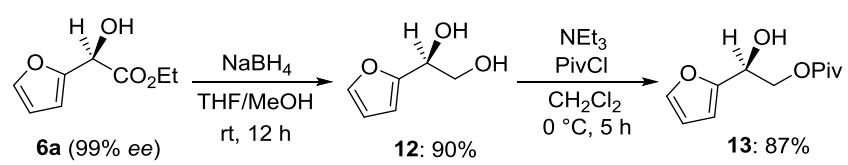

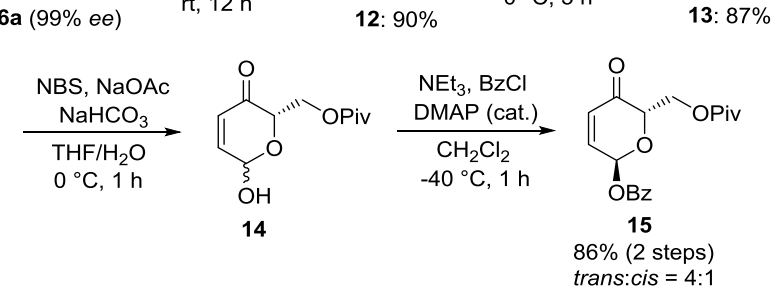

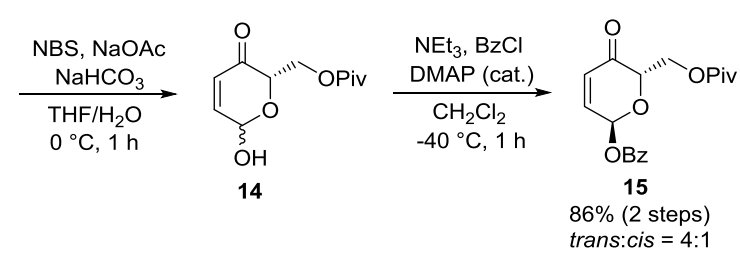

Scheme 4. Synthesis of a chiral pyranone from furan via an Achmatowicz rearrangement

The palladium-catalyzed methodology was then expanded for a two-directional reaction (Scheme 5). Following the FriedelCrafts alkylation of furan $\mathbf{4 a}$ with trifluoropyruvate $\mathbf{2}$ in the presence of $1 \mathbf{a}(2 \mathrm{~mol} \%)$ under the optimized reaction conditions, the reaction of glyoxylate $\mathbf{3}$ to intermediate $\mathbf{5 a}$ led to the twodirectional adduct $\mathbf{1 6}$ in $91 \%$ yield with $99 \%$ ee as a single diastereomer without the formation of the undesired meso type product.

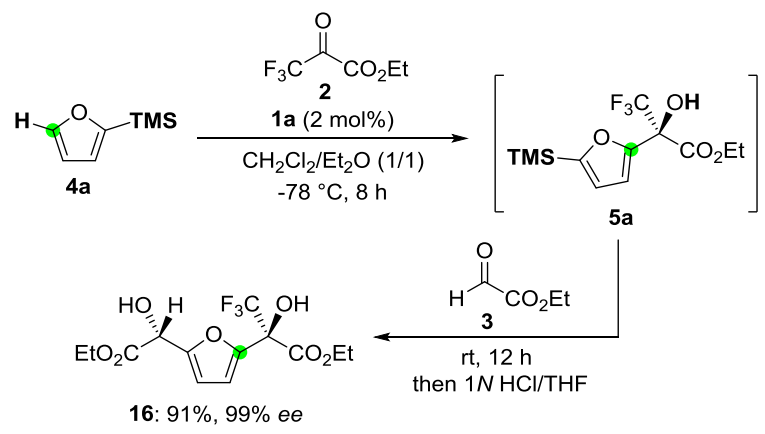

Scheme 5. Two-directional reaction of furan $4 a$ with ethyl trifluoropyruvate followed by ethyl glyoxylate

We also attempted to transform alkynyldiols, which can be readily synthesized from $\alpha$-hydroxy ketones, to $\alpha$-hydroxy esters by a one-pot operation (Scheme 6). Alkynyldiol 17 in toluene was converted in situ to 2-trimethylsilylated furan 11f via intramolecular cyclization by a cationic $\mathrm{Au}$ catalyst 19a. ${ }^{16,17}$ After the solution of ethyl glyoxylate 3 and dicationic Pd catalyst 1a in dichloromethane was transferred to a solution of $\mathbf{1 7}$, the Friedel-Crafts type reaction proceeded to produce the heteroarylated product 6 f in $57 \%$ yield and with $99 \%$ ee (Scheme 6, Eq. 1). In a similar manner to the glyoxylate system, intramolecular cyclization with alkynyldiol $\mathbf{1 8}$ by $\mathrm{Au}$ catalyst 19b followed by Friedel-Crafts reaction with trifluoropyruvate 2 using Pd catalyst 1a led to product $\mathbf{5 f}$ in $62 \%$ yield and with $99 \%$ ee (Scheme 6, Eq. 2). 
4

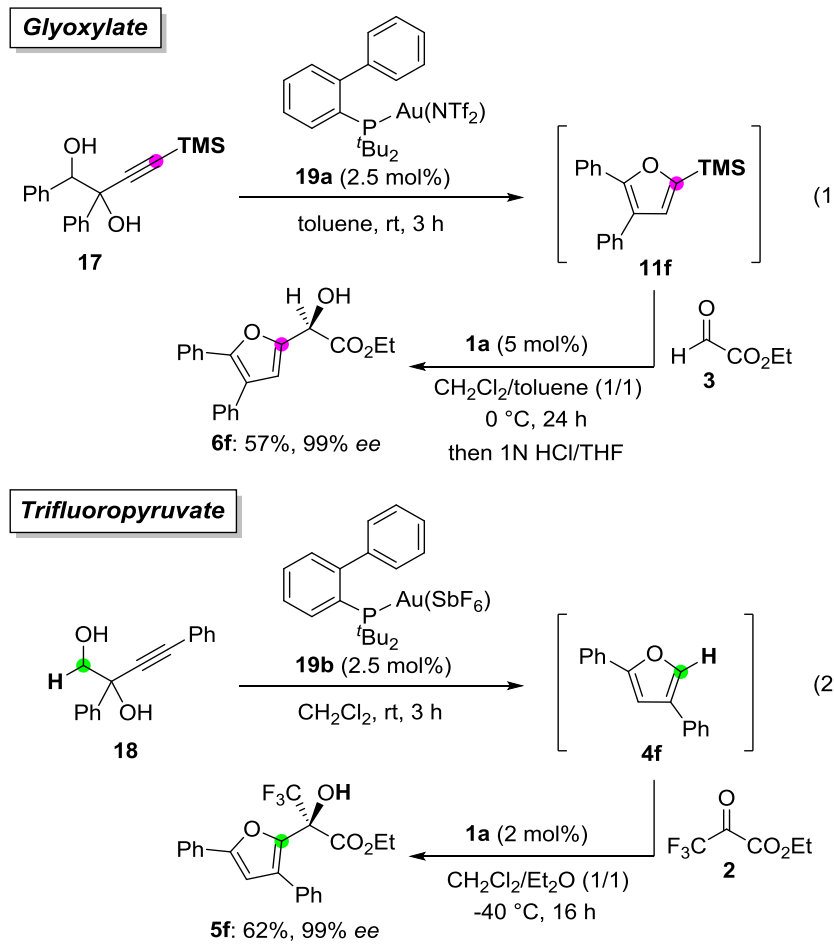

Scheme 6. Sequential catalytic intramolecular cyclization by an $A u$ catalyst followed by Friedel-Crafts type reactions by a Pd catalyst.

\section{Conclusion}

In conclusion, we have reported on the highly enantioselective Friedel-Crafts reactions of furan and thiophene derivatives with trifluoropyruvate by using dicationic palladium complexes as Lewis acid catalysts. Moreover, the Friedel-Crafts type reaction of 2-trimethylsilylated furan, thiophene, and pyrrole derivatives with glyoxylate led to a complete change of regioselectivity to give the heteroarylated products in high yields and enantioselectivities. Additionally, the catalytic intramolecular cyclization of alkynyldiols by a cationic gold catalyst followed by Friedel-Crafts type reactions with trifluoropyruvate or glyoxylate by a dicationic palladium catalyst gave the corresponding products with high enantioselectivities. The development of novel and practical reactions to provide optically active $\alpha$-fluoromethyl-substituted tertiary alcohols is currently ongoing in our laboratory.

\section{Experimental}

\subsection{General}

${ }^{1} \mathrm{H},{ }^{13} \mathrm{C}$, and ${ }^{19} \mathrm{~F}$ NMR spectra were measured on Bruker AV300M (300 MHz) spectrometers. The chemical shifts of ${ }^{1} \mathrm{H}$ NMR are expressed in parts per million relative to the singlet $(\delta$ $=7.26$ ) for $\mathrm{CDCl}_{3}$. The chemical shifts of ${ }^{13} \mathrm{C} \mathrm{NMR}$ are expressed in parts per million relative to the central line of the triplet $(\delta=77.0)$ for $\mathrm{CDCl}_{3}$. The chemical shifts of ${ }^{19} \mathrm{~F}$ NMR are expressed in parts per million relative to the singlet $(\delta=-63.24)$ for benzotrifluoride (BTF) as an internal standard. Optical rotations were measured on a JASCO P-1020. Mass spectra were measured on a JEOL JMS-T100CS (Accu-TOF) spectrometer. IR spectra were measured on a JASCO FT/IR-4200 spectrometer. High performance liquid chromatography (HPLC) was conducted on JASCO PU-980, LG-980-02, DG-980-50, MD-2010, and CO966 instrument equipped with model UV-975 spectrometers as an ultra violet light. Dichloromethane (dehydrate), toluene (dehydrate), and diethyl ether (dehydrate) were purchased from Kanto Chemical Co., Inc. Ethyl trifluoropyruvate was provided by Central Glass Co., Ltd. Silver hexafluoroantimonate and ethyl glyoxylate polymer form $(\sim 50 \mathrm{w} \%$ in toluene) were purchased from Aldrich. $\mathrm{PdCl}_{2}[(S)$-BINAP] was synthesized according to the literature procedure. ${ }^{18}$ Heteroarylsilanes $\mathbf{4 a},{ }^{19} \mathbf{1 1 b},{ }^{20} \mathbf{1 1 c},{ }^{21}$ $\mathbf{1 1 g},{ }^{22} \mathbf{1 1 h},{ }^{23} \mathbf{1 1},^{24}$ vinylsilane $7,{ }^{25}$ and dienylsilane $\mathbf{9}^{26}$ were also synthesized according to the literature procedures.

4.2. General procedure for the catalytic asymmetric FriedelCrafts reaction with trifluoropyruvate (Table 2)

To a solution of $\mathrm{PdCl}_{2}[(S)$-BINAP] $(3.2 \mathrm{mg}, 0.004 \mathrm{mmol})$ in $\mathrm{CH}_{2} \mathrm{Cl}_{2}(1.0 \mathrm{~mL})$ was added $\mathrm{AgSbF}_{6}(3.0 \mathrm{mg}, 0.0088 \mathrm{mmol})$ at room temperature under an argon atmosphere. After stirring for $30 \mathrm{~min}, \mathrm{Et}_{2} \mathrm{O}(1.0 \mathrm{~mL})$, ethyl trifluoropyruvate $2(32 \mu \mathrm{L}, 0.24$ $\mathrm{mmol})$, and heteroaryl compounds $4(0.2 \mathrm{mmol})$ were added at $78{ }^{\circ} \mathrm{C}$. The reaction mixture was stirred at $-78{ }^{\circ} \mathrm{C}$ for $8 \mathrm{~h}$, and then loaded directly onto a short silica-gel column (hexane/AcOEt $=1 / 1$ ) to remove the catalyst. Purification by a silica-gel chromatography (hexane/AcOEt $=9 / 1$ ) gave the corresponding alcohol product 5. The enantiomeric excess was determined by chiral HPLC analysis.

\subsection{1. $\quad(R)$-Ethyl 3,3,3-trifluoro-2-hydroxy-2-(5-} (trimethylsilyl)furan-2-yl)propanoate 5a

${ }^{1} \mathrm{H}$ NMR $\left(300 \mathrm{MHz}, \mathrm{CDCl}_{3}\right) \delta 0.25(\mathrm{~s}, 9 \mathrm{H}), 1.34(\mathrm{t}, J=7.2 \mathrm{~Hz}$, $3 \mathrm{H}), 4.36-4.44(\mathrm{~m}, 2 \mathrm{H}), 4.37(\mathrm{~s}, 1 \mathrm{H}), 6.57(\mathrm{~d}, J=3.3 \mathrm{~Hz}, 1 \mathrm{H})$, $6.60(\mathrm{~d}, J=3.3 \mathrm{~Hz}, 1 \mathrm{H}) ;{ }^{13} \mathrm{C} \mathrm{NMR}\left(75 \mathrm{MHz}, \mathrm{CDCl}_{3}\right) \delta-1.3,13.9$, $64.4,75.3\left(\mathrm{q}, J_{\mathrm{C}-\mathrm{F}}=31.5 \mathrm{~Hz}\right), 110.2,120.2,122.4\left(\mathrm{q}, J_{\mathrm{C}-\mathrm{F}}=284\right.$ $\mathrm{Hz}), 149.8,162.5,167.4 ;{ }^{19} \mathrm{~F}$ NMR $\left(282 \mathrm{MHz}, \mathrm{CDCl}_{3}\right) \delta$-76.2; FT-IR ( $\mathrm{KBr}$ pellet, $\mathrm{cm}^{-1}$ ) 3479, 2963, 1744, 1300, 1253, 1176, 1111, 1020, 932, 842; HRMS (ESI-TOF) calcd for $\mathrm{C}_{12} \mathrm{H}_{17} \mathrm{~F}_{3} \mathrm{NaO}_{4} \mathrm{Si}[\mathrm{M}+\mathrm{Na}]^{+}: 333.0746$, found: $333.0749 ;[\alpha]_{\mathrm{D}}{ }^{25}=-$ 15.2 (c 1.3, $\mathrm{CHCl}_{3}$ ), 99\% ee; HPLC (column, CHIRALPAK AD3 , Hexane $/ 2$-Propanol $=99 / 1$, flow rate $0.6 \mathrm{~mL} / \mathrm{min}, 20{ }^{\circ} \mathrm{C}$, detection UV $210 \mathrm{~nm}$ ) $t_{\mathrm{R}}$ of minor isomer $11.8 \mathrm{~min}, t_{\mathrm{R}}$ of major isomer $19.2 \mathrm{~min}$. The absolute configuration was determined to be $(R)$ in comparison with the specific rotation of the reported data. $^{5 \mathrm{~b}}$

\subsection{2. $\quad(R)$-Ethyl 3,3,3-trifluoro-2-(furan-2-yl)-2-} hydroxypropanoate $(5 \mathrm{~b})$

${ }^{1} \mathrm{H}$ NMR (300 MHz, $\left.\mathrm{CDCl}_{3}\right) \delta 1.35(\mathrm{t}, J=7.2 \mathrm{~Hz}, 3 \mathrm{H}), 4.37-4.46$ $(\mathrm{m}, 2 \mathrm{H}), 4.39(\mathrm{~s}, 1 \mathrm{H}), 6.42(\mathrm{~d}, J=3.0 \mathrm{~Hz}, 1 \mathrm{H}), 6.62(\mathrm{~d}, J=3.0$ $\mathrm{Hz}, 1 \mathrm{H}), 7.46$ (s, 1H); ${ }^{13} \mathrm{C}$ NMR $\left(75 \mathrm{MHz}, \mathrm{CDCl}_{3}\right) \delta$ 13.8, 64.7, $75.3\left(\mathrm{q}, J_{\mathrm{C}-\mathrm{F}}=30.5 \mathrm{~Hz}\right), 110.6,110.7,122.3\left(\mathrm{q}, J_{\mathrm{C}-\mathrm{F}}=284 \mathrm{~Hz}\right)$, 143.9, 145.9, 167.2; ${ }^{19} \mathrm{~F}$ NMR $\left(282 \mathrm{MHz}, \mathrm{CDCl}_{3}\right) \delta$-76.4; FT-IR $\left(\mathrm{KBr}\right.$ pellet, $\mathrm{cm}^{-1}$ ) 3473, 2992, 2946, 1746, 1372, 1308, 1249, 1189, 1155, 1015, 968, 751; HRMS (ESI-TOF) calcd for $\mathrm{C}_{9} \mathrm{H}_{9} \mathrm{~F}_{3} \mathrm{NaO}_{4}[\mathrm{M}+\mathrm{Na}]^{+}:$261.0351, found: 261.0363; $[\alpha]_{\mathrm{D}}{ }^{25}-20.3$ (c $0.80, \mathrm{CHCl}_{3}$ ), 99\% ee; HPLC (column, CHIRALPAK AD-3, Hexane/2-Propanol $=99 / 1$, flow rate $0.6 \mathrm{~mL} / \mathrm{min}, 20{ }^{\circ} \mathrm{C}$, detection UV $210 \mathrm{~nm}$ ) $t_{\mathrm{R}}$ of minor isomer $32.2 \mathrm{~min}, t_{\mathrm{R}}$ of major isomer $46.4 \mathrm{~min}$. The absolute configuration was tentatively assigned by analogy of the specific rotation.

4.2.3. (R)-Ethyl 3,3,3-trifluoro-2-hydroxy-2-(5-methylfuran-2yl)propanoate $(5 \mathrm{c})$

${ }^{1} \mathrm{H}$ NMR $\left(300 \mathrm{MHz}, \mathrm{CDCl}_{3}\right) \delta 1.35(\mathrm{t}, J=7.2 \mathrm{~Hz}, 3 \mathrm{H}), 2.29(\mathrm{~s}$, $3 \mathrm{H}), 4.35(\mathrm{~s}, 1 \mathrm{H}), 4.35-4.46(\mathrm{~m}, 2 \mathrm{H}), 5.99(\mathrm{~d}, J=3.0 \mathrm{~Hz}, 1 \mathrm{H})$, $6.47(\mathrm{~d}, J=3.0 \mathrm{~Hz}, 1 \mathrm{H}) ;{ }^{13} \mathrm{C} \mathrm{NMR}\left(75 \mathrm{MHz}, \mathrm{CDCl}_{3}\right) \delta 13.5$, $13.8,64.5,75.1\left(\mathrm{q}, J_{\mathrm{C}-\mathrm{F}}=30.5 \mathrm{~Hz}\right), 106.7,111.4,122.4\left(\mathrm{q}, J_{\mathrm{C}-\mathrm{F}}=\right.$ $284 \mathrm{~Hz}), 143.9,153.9,167.4 ;{ }^{19} \mathrm{~F}$ NMR $\left(282 \mathrm{MHz}, \mathrm{CDCl}_{3}\right) \delta$ 76.3; FT-IR (KBr pellet, $\mathrm{cm}^{-1}$ ) 3474, 2988, 2929, 1749, 1371, 1305, 1261, 1178, 1113, 1021, 791; HRMS (ESI-TOF) calcd for $\mathrm{C}_{10} \mathrm{H}_{11} \mathrm{~F}_{3} \mathrm{NaO}_{4}[\mathrm{M}+\mathrm{Na}]^{+}:$275.0507, found: $275.0495 ;[\alpha]_{\mathrm{D}}{ }^{25}=-$ 
10.7 (c 0.7, $\mathrm{CHCl}_{3}$ ), 94\% ee; HPLC (column, CHIRALPAK AD3 , Hexane/2-Propanol $=99 / 1$, flow rate $0.6 \mathrm{~mL} / \mathrm{min}, 20{ }^{\circ} \mathrm{C}$, detection UV $210 \mathrm{~nm}$ ) $t_{\mathrm{R}}$ of minor isomer $20.7 \mathrm{~min}, t_{\mathrm{R}}$ of major isomer $22.7 \mathrm{~min}$. The absolute configuration was tentatively assigned by analogy of the specific rotation.

\subsection{4. (R)-Ethyl 3,3,3-trifluoro-2-hydroxy-2-(5-phenylfuran-2-} yl)propanoate $(5 d)$

${ }^{1} \mathrm{H}$ NMR $\left(300 \mathrm{MHz}, \mathrm{CDCl}_{3}\right) \delta 1.37(\mathrm{t}, J=7.2 \mathrm{~Hz}, 3 \mathrm{H}), 4.42-4.50$ $(\mathrm{m}, 2 \mathrm{H}), 4.43(\mathrm{~s}, 1 \mathrm{H}), 6.65(\mathrm{~d}, J=3.6 \mathrm{~Hz}, 1 \mathrm{H}), 6.69(\mathrm{~d}, J=3.6$ $\mathrm{Hz}, 1 \mathrm{H}), 7.29-7.39$ (m, 3H), $7.66(\mathrm{~d}, J=5.4 \mathrm{~Hz}, 2 \mathrm{H}) ;{ }^{13} \mathrm{C}$ NMR $\left(75 \mathrm{MHz}, \mathrm{CDCl}_{3}\right) \delta 13.9,64.7,75.1\left(\mathrm{q}, J_{\mathrm{C}-\mathrm{F}}=32.0 \mathrm{~Hz}\right), 105.7$, $112.5,122.4\left(\mathrm{q}, J_{\mathrm{C}-\mathrm{F}}=284 \mathrm{~Hz}\right), 124.0,128.1,128.7,130.0,145.2$, 155.3, 167.3; ${ }^{19} \mathrm{~F}$ NMR $\left(282 \mathrm{MHz}, \mathrm{CDCl}_{3}\right) \delta$-76.2; FT-IR $(\mathrm{KBr}$ pellet, $\mathrm{cm}^{-1}$ ) 3475, 2986, 2933, 1745, 1486, 1371, 1304, 1179, $1115,1017,760,691$; HRMS (ESI-TOF) calcd for $\mathrm{C}_{15} \mathrm{H}_{13} \mathrm{~F}_{3} \mathrm{NaO}_{4}$ $[\mathrm{M}+\mathrm{Na}]^{+}: 337.0664$, found: $337.0654 ;[\alpha]_{\mathrm{D}}{ }^{25}=-35.2(c 1.2$, $\mathrm{CHCl}_{3}$ ), 99\% ee; HPLC (column, CHIRALPAK AD-3, Hexane $/ 2$-Propanol $=99 / 1$, flow rate $0.6 \mathrm{~mL} / \mathrm{min}, 20{ }^{\circ} \mathrm{C}$, detection UV $210 \mathrm{~nm}$ ) $t_{\mathrm{R}}$ of minor isomer $29.4 \mathrm{~min}, t_{\mathrm{R}}$ of major isomer $42.4 \mathrm{~min}$. The absolute configuration was tentatively assigned by analogy of the specific rotation.

\subsection{5. $\quad(R)$-Ethyl 2-(5-((tert- butyldiphenylsilyl)oxy)methyl)furan-2-yl)-3,3,3-trifluoro-2- hydroxypropanoate (5e)}

${ }^{1} \mathrm{H}$ NMR $\left(300 \mathrm{MHz}, \mathrm{CDCl}_{3}\right) \delta 1.04(\mathrm{~s}, 9 \mathrm{H}), 1.31(\mathrm{t}, J=7.2 \mathrm{~Hz}$, $3 \mathrm{H}), 4.32(\mathrm{~s}, 1 \mathrm{H}), 4.32-4.47(\mathrm{~m}, 2 \mathrm{H}), 4.62(\mathrm{~s}, 2 \mathrm{H}), 6.17(\mathrm{~d}, J=$ $3.3 \mathrm{~Hz}, 1 \mathrm{H}), 6.52(\mathrm{~d}, J=3.3 \mathrm{~Hz}, 1 \mathrm{H}), 7.36-7.44(\mathrm{~m}, 6 \mathrm{H}), 7.68(\mathrm{~d}$, $J=7.5 \mathrm{~Hz}, 4 \mathrm{H}) ;{ }^{13} \mathrm{C}$ NMR $\left(75 \mathrm{MHz}, \mathrm{CDCl}_{3}\right) \delta 13.9,19.2,26.6$, $58.7,64.6,75.1\left(\mathrm{q}, J_{\mathrm{C}-\mathrm{F}}=32.0 \mathrm{~Hz}\right), 108.2,111.2,122.3\left(\mathrm{q}, J_{\mathrm{C}-\mathrm{F}}=\right.$ $284 \mathrm{~Hz}), 127.7,129.8,133.1,135.6,145.2,155.6,167.3 ;{ }^{19} \mathrm{~F}$ NMR $\left(282 \mathrm{MHz}, \mathrm{CDCl}_{3}\right) \delta$-76.6; FT-IR ( $\mathrm{KBr}$ pellet, $\left.\mathrm{cm}^{-1}\right) 3479$, 2957, 2932, 2858, 1745, 1428, 1308, 1178, 1111, 1019, 823,703; HRMS (ESI-TOF) calcd for $\mathrm{C}_{26} \mathrm{H}_{29} \mathrm{~F}_{3} \mathrm{KO}_{5} \mathrm{Si}[\mathrm{M}+\mathrm{K}]^{+}$: 545.1373, found: $545.1355 ;[\alpha]_{\mathrm{D}}{ }^{25}=-19.0\left(c 0.7, \mathrm{CHCl}_{3}\right), 99 \% e e$; HPLC (column, CHIRALCEL OD-3, Hexane/2-Propanol = 99/1, flow rate $0.6 \mathrm{~mL} / \mathrm{min}, 20^{\circ} \mathrm{C}$, detection UV $210 \mathrm{~nm}$ ) $t_{\mathrm{R}}$ of minor isomer $18.4 \mathrm{~min}, t_{\mathrm{R}}$ of major isomer $20.1 \mathrm{~min}$. The absolute configuration was tentatively assigned by analogy of the specific rotation.

\subsection{6. (R)-Ethyl 2-(3,5-diphenylfuran-2-yl)-3,3,3-trifluoro-2-} hydroxypropanoate $\mathbf{5 f}$

${ }^{1} \mathrm{H}$ NMR (300 MHz, $\mathrm{CDCl}_{3}$ ) $\delta 1.05(\mathrm{t}, J=7.2 \mathrm{~Hz}, 3 \mathrm{H}), 3.61-3.67$ $(\mathrm{m}, 1 \mathrm{H}), 4.01-4.07(\mathrm{~m}, 1 \mathrm{H}), 4.52(\mathrm{~s}, 1 \mathrm{H}), 6.76(\mathrm{~s}, 1 \mathrm{H}), 7.34-7.47$ $(\mathrm{m}, 8 \mathrm{H}), 7.75(\mathrm{~d}, J=7.8 \mathrm{~Hz}, 2 \mathrm{H}) ;{ }^{13} \mathrm{C}$ NMR $\left(75 \mathrm{MHz}, \mathrm{CDCl}_{3}\right) \delta$ $13.4,64.4,75.3\left(\mathrm{q}, J_{\mathrm{C}-\mathrm{F}}=31.0 \mathrm{~Hz}\right), 108.3,122.6\left(\mathrm{q}, J_{\mathrm{C}-\mathrm{F}}=284\right.$ $\mathrm{Hz}), 124.1,127.9,128.2$ (2C), 128.8, 129.1, 129.4, 129.7, 132.3, 140.6, 153.7, 167.3; ${ }^{19} \mathrm{~F}$ NMR $\left(282 \mathrm{MHz}, \mathrm{CDCl}_{3}\right) \delta$-74.3; FT-IR $\left(\mathrm{KBr}\right.$ pellet, $\left.\mathrm{cm}^{-1}\right)$ 3465, 3059, 2985, 1743, 1299, 1221, 1167, 1143, 916, 760, 700, 691, 673; HRMS (ESI-TOF) calcd for $\mathrm{C}_{21} \mathrm{H}_{17} \mathrm{~F}_{3} \mathrm{NaO}_{4}[\mathrm{M}+\mathrm{Na}]^{+}: 413.0977$, found: 413.0991; $[\alpha]_{\mathrm{D}}{ }^{25}-23.3$ (c 1.3, $\mathrm{CHCl}_{3}$ ), 99\% ee; HPLC (column, CHIRALPAK AD-3, Hexane $/ 2$-Propanol $=99 / 1$, flow rate $0.6 \mathrm{~mL} / \mathrm{min}, 20{ }^{\circ} \mathrm{C}$, detection UV $210 \mathrm{~nm}) t_{\mathrm{R}}$ of major isomer $33.9 \mathrm{~min}, t_{\mathrm{R}}$ of minor isomer $55.7 \mathrm{~min}$. The absolute configuration was tentatively assigned by analogy of the specific rotation.

\subsection{7. $\quad(R)$-Ethyl 3,3,3-trifluoro-2-hydroxy-2-(thiophen-2-} yl)propanoate $5 \mathrm{~h}$

${ }^{1} \mathrm{H}$ NMR $\left(300 \mathrm{MHz}, \mathrm{CDCl}_{3}\right) \delta 1.39(\mathrm{t}, J=7.2 \mathrm{~Hz}, 3 \mathrm{H}), 4.38-4.48$ $(\mathrm{m}, 2 \mathrm{H}), 4.63(\mathrm{~s}, 1 \mathrm{H}), 7.05(\mathrm{dd}, J=5.1,3.6 \mathrm{~Hz}, 1 \mathrm{H}) 7.36-7.39$ $(\mathrm{m}, 2 \mathrm{H}) ;{ }^{13} \mathrm{C}$ NMR $\left(75 \mathrm{MHz}, \mathrm{CDCl}_{3}\right) \delta 13.8,64.8,76.7\left(\mathrm{q}, J_{\mathrm{C}-\mathrm{F}}=\right.$ $31.2 \mathrm{~Hz}), 122.4\left(\mathrm{q}, J_{\mathrm{C}-\mathrm{F}}=284 \mathrm{~Hz}\right), 127.2,127.3,127.5,135.9$, $168.0 ;{ }^{19} \mathrm{~F} \mathrm{NMR}\left(282 \mathrm{MHz}, \mathrm{CDCl}_{3}\right) \delta$-78.0; FT-IR (KBr pellet, $\left.\mathrm{cm}^{-1}\right) 3469,2988,2942,1743,1306,1236,1190,1166,1011,856$, 711; HRMS (ESI-TOF) calcd for $\mathrm{C}_{9} \mathrm{H}_{9} \mathrm{~F}_{3} \mathrm{NaO}_{3} \mathrm{~S}[\mathrm{M}+\mathrm{Na}]^{+}$: 277.0122, found: 277.0116; $[\alpha]_{\mathrm{D}}^{25}=-14.5\left(c 1.4, \mathrm{CHCl}_{3}\right), 94 \%$ $e e$; HPLC (column, CHIRALPAK AD-3, Hexane/2-Propanol = $99 / 1$, flow rate $0.6 \mathrm{~mL} / \mathrm{min}, 20{ }^{\circ} \mathrm{C}$, detection UV $\left.210 \mathrm{~nm}\right) t_{\mathrm{R}}$ of minor isomer $17.2 \mathrm{~min}, t_{\mathrm{R}}$ of major isomer $22.8 \mathrm{~min}$. The absolute configuration was tentatively assigned by analogy of the specific rotation.

\subsection{8. (R)-Ethyl 3,3,3-trifluoro-2-hydroxy-2-(5-} methylthiophen-2-yl)propanoate $5 \mathbf{i}$

${ }^{1} \mathrm{H}$ NMR $\left(300 \mathrm{MHz}, \mathrm{CDCl}_{3}\right) \delta 1.39(\mathrm{t}, J=7.2 \mathrm{~Hz}, 3 \mathrm{H}), 2.47(\mathrm{~s}$, $3 \mathrm{H}), 4.38-4.47(\mathrm{~m}, 2 \mathrm{H}), 4.55(\mathrm{~s}, 1 \mathrm{H}), 6.69(\mathrm{~d}, J=3.6 \mathrm{~Hz}, 1 \mathrm{H})$ $7.16(\mathrm{~d}, J=3.6 \mathrm{~Hz}, 1 \mathrm{H}) ;{ }^{13} \mathrm{C} \mathrm{NMR}\left(75 \mathrm{MHz}, \mathrm{CDCl}_{3}\right) \delta 13.8,16.1$, 64.6, $76.6\left(\mathrm{q}, J_{\mathrm{C}-\mathrm{F}}=32.4 \mathrm{~Hz}\right), 122.4\left(\mathrm{q}, J_{\mathrm{C}-\mathrm{F}}=284 \mathrm{~Hz}\right), 125.5$, 127.5, 132.9, 141.9, 168.2; ${ }^{19} \mathrm{~F}$ NMR $\left(282 \mathrm{MHz} \mathrm{CDCl}_{3}\right) \delta$-78.0; FT-IR (KBr pellet, $\mathrm{cm}^{-1}$ ) 3475, 2987, 2928, 1741, 1372, 1301, 1242, 1188, 1131, 1014, 805, 671; HRMS (ESI-TOF) calcd for $\mathrm{C}_{10} \mathrm{H}_{11} \mathrm{~F}_{3} \mathrm{NaO}_{3} \mathrm{~S}[\mathrm{M}+\mathrm{Na}]^{+}: 291.0279$, found: $291.0270 ;[\alpha]_{\mathrm{D}}{ }^{25}=-$ 10.6 ( $c$ 0.8, $\mathrm{CHCl}_{3}$ ), 97\% ee; HPLC (column, CHIRALPAK AD3 , Hexane $/ 2$-Propanol $=99 / 1$, flow rate $0.6 \mathrm{~mL} / \mathrm{min}, 20{ }^{\circ} \mathrm{C}$, detection UV $210 \mathrm{~nm}$ ) $t_{\mathrm{R}}$ of minor isomer $18.6 \mathrm{~min}, t_{\mathrm{R}}$ of major isomer $23.0 \mathrm{~min}$. The absolute configuration was tentatively assigned by analogy of the specific rotation.

4.2.9. tert-Butyl 2-(3-ethoxy-1,1,1-trifluoro-2-hydroxy-3oxopropan-2-yl)-1H-pyrrole-1-carboxylate $5 \mathbf{j}$

${ }^{1} \mathrm{H}$ NMR (300 MHz, $\mathrm{CDCl}_{3}$ ) 2-substituted product: $\delta 1.28(\mathrm{t}, J=$ $7.2 \mathrm{~Hz}, 3 \mathrm{H}), 1.58(\mathrm{~s}, 9 \mathrm{H}), 4.26-4.33(\mathrm{~m}, 2 \mathrm{H}), 4.27(\mathrm{~s}, 1 \mathrm{H}), 6.43$ $(\mathrm{m}, 1 \mathrm{H}), 6.56(\mathrm{~m}, 1 \mathrm{H}), 7.48(\mathrm{t}, J=2.1 \mathrm{~Hz}, 1 \mathrm{H}) ; 3$-substituted product: $\delta 1.38(\mathrm{t}, J=7.2 \mathrm{~Hz}, 3 \mathrm{H}), 1.61(\mathrm{~s}, 9 \mathrm{H}), 4.38-4.46(\mathrm{~m}$, $2 \mathrm{H}), 5.18(\mathrm{~s}, 1 \mathrm{H}), 6.19(\mathrm{t}, J=3.6 \mathrm{~Hz}, 1 \mathrm{H}) 7.22-7.26(\mathrm{~m}, 2 \mathrm{H}) ;{ }^{13} \mathrm{C}$ NMR (75 MHz, $\left.\mathrm{CDCl}_{3}\right)$ a mixture of 2- and 3- substituted products: $\delta 13.84,13.88,27.8,27.9,63.2,64.2,75.3$ (q, $J_{\mathrm{C}-\mathrm{F}}=$ $31.5 \mathrm{~Hz}), 76.4\left(\mathrm{q}, J_{\mathrm{C}-\mathrm{F}}=31.3 \mathrm{~Hz}\right), 84.3,85.4,110.4,110.9,116.2$, $119.6,120.2,120.5,122.9\left(\mathrm{q}, J_{\mathrm{C}-\mathrm{F}}=284 \mathrm{~Hz}\right), 123.1\left(\mathrm{q}, J_{\mathrm{C}-\mathrm{F}}=286\right.$ $\mathrm{Hz}), 148.3,149.9,167.4,168.8 ;{ }^{19} \mathrm{~F}$ NMR $\left(282 \mathrm{MHz}, \mathrm{CDCl}_{3}\right) 2-$ substituted product: $\delta$-78.4, 3-substituted product: $\delta$-74.0; FT-IR ( $\mathrm{KBr}$ pellet, $\mathrm{cm}^{-1}$ ) 3482, 2984, 2938, 1749, 1371, 1231, 1175, 1141, 978, 846, 772; HRMS (ESI-TOF) calcd for $\mathrm{C}_{14} \mathrm{H}_{18} \mathrm{~F}_{3} \mathrm{KNO}_{5}$ [M+K]: 376.0774, found: 376.0764 .

4.2.10. (R)-Ethyl 3,3,3-trifluoro-2-hydroxy-2-(1H-pyrrol-2yl)propanoate $5 \mathbf{k}$

${ }^{1} \mathrm{H}$ NMR $\left(300 \mathrm{MHz}, \mathrm{CDCl}_{3}\right) \delta 1.40(\mathrm{t}, J=7.2 \mathrm{~Hz}, 3 \mathrm{H}), 4.41-4.47$ $(\mathrm{m}, 2 \mathrm{H}), 6.24(\mathrm{~d}, J=3.3 \mathrm{~Hz}, 1 \mathrm{H}) 6.48(\mathrm{~s}, 1 \mathrm{H}), 6.83(\mathrm{~s}, 1 \mathrm{H}), 8.79$ (br, $1 \mathrm{H}) ;{ }^{13} \mathrm{C}$ NMR $\left(75 \mathrm{MHz}, \mathrm{CDCl}_{3}\right) \delta 13.8,64.6,75.1\left(\mathrm{q}, J_{\mathrm{C}-\mathrm{F}}=\right.$ $32.5 \mathrm{~Hz}), 108.9,109.3,118.8,121.7,122.5$ (q, $\left.J_{\mathrm{C}-\mathrm{F}}=284 \mathrm{~Hz}\right)$, 168.0; ${ }^{19} \mathrm{~F}$ NMR $\left(282 \mathrm{MHz}, \mathrm{CDCl}_{3}\right) \delta$-78.4; FT-IR (KBr pellet, $\mathrm{cm}^{-1}$ ) 3435, 3367, 3123, 2991, 1738, 1265, 1226, 1182, 817, 746; HRMS (ESI-TOF) calcd for $\mathrm{C}_{9} \mathrm{H}_{9} \mathrm{~F}_{3} \mathrm{NO}_{3}[\mathrm{M}-\mathrm{H}]$ : : 236.0535, found: $236.0528 ;[\alpha]_{\mathrm{D}}{ }^{25}=-8.6\left(c 0.8, \mathrm{CHCl}_{3}\right), 37 \%$ ee; HPLC (column, CHIRALPAK AD-3, Hexane/2-Propanol = 97/3, flow rate $0.6 \mathrm{~mL} / \mathrm{min}, 20{ }^{\circ} \mathrm{C}$, detection UV $\left.210 \mathrm{~nm}\right) t_{\mathrm{R}}$ of minor isomer $38.9 \mathrm{~min}, t_{\mathrm{R}}$ of major isomer $45.7 \mathrm{~min}$. The absolute configuration was tentatively assigned by analogy of the specific rotation.

4.3. Procedure for the catalytic asymmetric alkenylation and dienylation with trifluoropyruvate (Scheme 3)

To a solution of $\mathrm{PdCl}_{2}[(S)$-BINAP $](8.0 \mathrm{mg}, 0.01 \mathrm{mmol})$ in $\mathrm{CH}_{2} \mathrm{Cl}_{2}(2.0 \mathrm{~mL})$ was added $\mathrm{AgSbF}_{6}(7.6 \mathrm{mg}, 0.022 \mathrm{mmol})$ at room temperature under an argon atmosphere. After stirring for $30 \mathrm{~min}$, ethyl trifluoropyruvate $2(53 \mu \mathrm{L}, 0.4 \mathrm{mmol})$ and vinylsilane 8 or dienylsilane $9(0.2 \mathrm{mmol})$ were added at $0{ }^{\circ} \mathrm{C}$. The reaction mixture was stirred at $0{ }^{\circ} \mathrm{C}$ for $24 \mathrm{~h}$, and then loaded 
directly onto a short silica-gel column (hexane/AcOEt $=1 / 1)$ to remove the catalyst. To a solution of the crude product in THF $(1.0 \mathrm{~mL})$ was added $6 \mathrm{M} \mathrm{HCl}(1.0 \mathrm{~mL})$. After stirring for $4 \mathrm{~h}$ at room temperature, saturated aqueous $\mathrm{NaHCO}_{3}(2.0 \mathrm{~mL})$ was added, and then the mixture was extracted with ether. The organic layer was washed with brine, dried over $\mathrm{Na}_{2} \mathrm{SO}_{4}$, and evaporated under reduced pressure. Purification by a short silicagel chromatography (hexane/AcOEt = 5/1) gave the corresponding alcohol products. The enantiomeric excess was determined by chiral HPLC analysis.

\subsection{1. $\quad(E, R)$-2-Hydroxy-4-phenyl-2-trifluoromethyl-but-3- enoic acid ethyl ester 8}

${ }^{1} \mathrm{H}$ NMR (300 MHz, $\left.\mathrm{CDCl}_{3}\right) \delta 1.36(\mathrm{t}, J=7.2 \mathrm{~Hz}, 3 \mathrm{H}), 4.14(\mathrm{~s}$, $1 \mathrm{H}), 4.30-4.51(\mathrm{~m}, 2 \mathrm{H}), 6.35(\mathrm{~d}, J=15.9 \mathrm{~Hz}, 1 \mathrm{H}), 7.12(\mathrm{~d}, J=$ $15.9 \mathrm{~Hz}, 1 \mathrm{H}), 7.30-7.38(\mathrm{~m}, 3 \mathrm{H}), 7.43-7.45(\mathrm{~m}, 2 \mathrm{H}) ;{ }^{13} \mathrm{C}$ NMR $\left(75 \mathrm{MHz}, \mathrm{CDCl}_{3}\right) \delta 13.9,64.4,77.1\left(\mathrm{q}, J_{\mathrm{C}-\mathrm{F}}=31.0 \mathrm{~Hz}\right), 119.3$, $122.8\left(\mathrm{q}, J_{\mathrm{C}-\mathrm{F}}=286 \mathrm{~Hz}\right), 127.1,128.69,128.72,134.8,135.2$, $168.9 ;{ }^{19} \mathrm{~F}$ NMR $\left(282 \mathrm{MHz}, \mathrm{CDCl}_{3}\right) \delta$-78.07; FT-IR (neat, $\mathrm{cm}^{-1}$ ) 3484, 3063, 3024, 2988, 2932, 1741, 1650, 1448, 1368, 1305, 1166, 1043, 856; HRMS (ESI-TOF) calcd for $\mathrm{C}_{13} \mathrm{H}_{13} \mathrm{~F}_{3} \mathrm{NaO}_{3}$ $[\mathrm{M}+\mathrm{Na}]^{+}:$297.0715, found: 297.0710; $[\alpha]_{\mathrm{D}}{ }^{23}=-68.6(c 1.6$, $\mathrm{CHCl}_{3}$ ), 98\% ee; HPLC (column, CHIRALPAK AD-H, Hexane/2-Propanol $=97 / 3$, flow rate $0.6 \mathrm{~mL} / \mathrm{min}, 20{ }^{\circ} \mathrm{C}$, detection UV $254 \mathrm{~nm}$ ) $t_{\mathrm{R}}$ of major isomer $21.2 \mathrm{~min}, t_{\mathrm{R}}$ of minor isomer $25.6 \mathrm{~min}$. The absolute configuration was tentatively assigned by analogy of the specific rotation. ${ }^{10 \mathrm{~d}}$

\subsection{2. $\quad(R, 3 E, 5 E)$-Ethyl 2-hydroxy-6-phenyl-2- (trifluoromethyl)hexa-3,5-dienoate (10)}

${ }^{1} \mathrm{H}$ NMR (300 MHz, CDCl $) \delta 1.37(\mathrm{t}, J=7.2 \mathrm{~Hz}, 3 \mathrm{H}), 4.07$ (s, $1 \mathrm{H}), 4.32-4.46(\mathrm{~m}, 2 \mathrm{H}), 5.96(\mathrm{~d}, J=4.1 \mathrm{~Hz}, 1 \mathrm{H}), 6.67-7.00(\mathrm{~m}$, 3H), 7.23-7.43 (m, 5H); ${ }^{13} \mathrm{C}$ NMR (75 MHz, $\left.\mathrm{CDCl}_{3}\right) \delta 13.9,64.3$, $76.3\left(\mathrm{q}, J_{\mathrm{C}-\mathrm{F}}=33.8 \mathrm{~Hz}\right), 122.7,122.9\left(\mathrm{q}, J_{\mathrm{C}-\mathrm{F}}=282 \mathrm{~Hz}\right), 126.6$, 126.7, 128.2, 128.7, 134.9, 135.9, 136.6, 168.9; FT-IR (neat, $\mathrm{cm}^{-}$ $\left.{ }^{1}\right) 3490,2919,2845,1731,1634,1589,1453,1311,1283,1011$; HRMS (APCI-TOF) calcd for $\mathrm{C}_{15} \mathrm{H}_{15} \mathrm{NaF}_{3} \mathrm{NaO}_{3} \quad[\mathrm{M}+\mathrm{Na}]^{+}$: 323.0871, found: 323.0866; $[\alpha]_{\mathrm{D}}{ }^{25}=-33.7\left(\right.$ c 2.16, $\left.\mathrm{CHCl}_{3}\right), 99 \%$ $e e$; HPLC (column, CHIRALCEL OD-H, Hexane/2-Propanol = $97 / 3$, flow rate $0.6 \mathrm{~mL} / \mathrm{min}, 20^{\circ} \mathrm{C}$, detection UV $254 \mathrm{~nm}$ ) $t_{\mathrm{R}}$ of minor isomer $19.8 \mathrm{~min}, t_{\mathrm{R}}$ of major isomer $27.1 \mathrm{~min}$. The absolute configuration was tentatively assigned by analogy of the specific rotation. ${ }^{10 \mathrm{~d}}$

\subsection{Synthesis of 2-trimethylsilylated heteroarylsilanes}

General procedure A: To a solution of the corresponding heteroaryl compound $(5.0 \mathrm{mmol})$ in THF $(20 \mathrm{~mL})$ was slowly added $n$-BuLi in hexane $(1.6 \mathrm{M})(3.44 \mathrm{~mL}, 5.5 \mathrm{mmol})$ at $-78{ }^{\circ} \mathrm{C}$. The mixture was then allowed to warm up slowly to room temperature and stirred for $1 \mathrm{~h}$, after which chlorotrimethylsilane $(694 \mu \mathrm{L}, 5.5 \mathrm{mmol})$ was added to the reaction mixture at $-78^{\circ} \mathrm{C}$. The reaction mixture was allowed to warm slowly to room temperature, stirred for $4 \mathrm{~h}$, and then quenched with saturated aqueous $\mathrm{NH}_{4} \mathrm{Cl}$. The organic layer was separated, and the aqueous layer was extracted with ether. The combined organic layer was washed with brine, dried over $\mathrm{Na}_{2} \mathrm{SO}_{4}$, and evaporated under reduced pressure. The residue was purified by column chromatography on silica gel to afford the corresponding heteroarylsilane products.

General procedure B: Freshly distilled diisopropylamine $(0.94$ $\mathrm{mL}, 6.6 \mathrm{mmol}, 1.1$ eq. $)$ in $\mathrm{THF}(10 \mathrm{~mL})$ was cooled to $-78{ }^{\circ} \mathrm{C}$. Next, $n$-BuLi in hexane $(1.6 \mathrm{M})(4.12 \mathrm{~mL}, 6.6 \mathrm{mmol}, 1.1 \mathrm{eq})$ was slowly added to the THF solution at $-78{ }^{\circ} \mathrm{C}$, and the mixture was allowed to warm up to $0{ }^{\circ} \mathrm{C}$. The mixture was stirred for $1 \mathrm{~h}$ at 0 ${ }^{\circ} \mathrm{C}$ before recooling to $-78{ }^{\circ} \mathrm{C}$. A solution of 1-tert-butyl pyrrole carboxylate derivative $(6.0 \mathrm{mmol}, 1.0$ eq. $)$ in THF $(20 \mathrm{~mL})$ was cooled to $-78{ }^{\circ} \mathrm{C}$. The LDA solution was added via cannula over 20 min at $-78{ }^{\circ} \mathrm{C}$. The mixture was stirred at $-78{ }^{\circ} \mathrm{C}$ for a further $6 \mathrm{~h}$ before trimethylsilylchloride $(1.0 \mathrm{~mL}, 7.2 \mathrm{mmol}, 1.2 \mathrm{eq}$.) was added. The reaction mixture was allowed to warm up slowly to 0 ${ }^{\circ} \mathrm{C}$ and stirred for $10 \mathrm{~h}$. The reaction mixture was poured into ice cold water. The organic layer was separated, and the aqueous layer was extracted with ethyl acetate. The combined organic layer was washed with brine, dried over $\mathrm{MgSO}_{4}$, and evaporated under reduced pressure. The residue was purified by column chromatography on silica gel to afford the corresponding heteroarylsilane products.

4.4.1. yl)methoxy)silane 11d

The title compound was synthesized from $4 \mathbf{e}$ according to General Procedure A (yield 60\%). ${ }^{1} \mathrm{H}$ NMR $\left(300 \mathrm{MHz}, \mathrm{CDCl}_{3}\right) \delta$ 0.29 (s, 9H), $1.10(\mathrm{~s}, 9 \mathrm{H}), 4.73(\mathrm{~s}, 2 \mathrm{H}), 6.18(\mathrm{~d}, J=3.3 \mathrm{~Hz}, 1 \mathrm{H})$, $6.56(\mathrm{~d}, J=3.0 \mathrm{~Hz}, 1 \mathrm{H}), 7.38-7.70(\mathrm{~m}, 10 \mathrm{H}) ;{ }^{13} \mathrm{C} \mathrm{NMR}(75 \mathrm{MHz}$, $\left.\mathrm{CDCl}_{3}\right) \delta-1.6,19.3,26.7,59.1,107.2,120.1,127.6,129.6,133.5$, 135.6, 158.2, 159.7; FT-IR (neat, $\mathrm{cm}^{-1}$ ) 3074, 2962, 2936, 2857, 1476, 1427, 1250, 1115, 908, 833, 735; HRMS (ESI-TOF) calcd for $\mathrm{C}_{24} \mathrm{H}_{32} \mathrm{NaO}_{2} \mathrm{Si}_{2}[\mathrm{M}+\mathrm{Na}]^{+}:$431.1838, found: 431.1822 .

\subsection{2. (3,5-Diphenylfuran-2-yl)trimethylsilane 11e}

The title compound was synthesized from $\mathbf{4 f}$ according to General Procedure A (yield 82\%). ${ }^{1} \mathrm{H}$ NMR $\left(300 \mathrm{MHz}, \mathrm{CDCl}_{3}\right) \delta$ 0.33 (s, 9H), 6.83 (s, 1H), 7.28-7.49 (m, 8H), 7.77 (d, $J=2.4 \mathrm{~Hz}$, $2 \mathrm{H}) ;{ }^{13} \mathrm{C}$ NMR $\left(75 \mathrm{MHz}, \mathrm{CDCl}_{3}\right) \delta-1.6,114.0,123.9,126.3$, $126.9,127.4,128.3,128.5,128.6,131.5,134.7,152.4,159.2$; FTIR (neat, $\mathrm{cm}^{-1}$ ) 3059, 2957, 1604, 1502, 1446, 1269, 1246, 942, 840, 761, 698; HRMS (ESI-TOF) calcd for $\mathrm{C}_{19} \mathrm{H}_{21} \mathrm{OSi}[\mathrm{M}+\mathrm{H}]^{+}$: 293.1361, found: 293.1348 .

\subsubsection{Trimethyl(5-phenylthiophen-2-yl)silane 11i}

The title compound was synthesized from 2-phenylthiophene according to General Procedure A (yield 83\%). ${ }^{1} \mathrm{H}$ NMR (300 $\left.\mathrm{MHz}, \mathrm{CDCl}_{3}\right) \delta 0.31(\mathrm{~s}, 9 \mathrm{H}), 7.18(\mathrm{~d}, J=3.3 \mathrm{~Hz}, 1 \mathrm{H}), 7.23(\mathrm{~d}, J$ $=4.8 \mathrm{~Hz}, 1 \mathrm{H}), 7.31-7.37(\mathrm{~m}, 3 \mathrm{H}), 7.59(\mathrm{~d}, J=7.5 \mathrm{~Hz}, 2 \mathrm{H}) ;{ }^{13} \mathrm{C}$ NMR $\left(75 \mathrm{MHz}, \mathrm{CDCl}_{3}\right) \delta-0.09,124.4,126.0,127.4,128.8$, 134.4, 135.0, 140.1, 149.6; FT-IR (neat, $\mathrm{cm}^{-1}$ ) 3059, 2954, 2894, 1596, 1483, 1430, 1246, 1078, 995, 840, 754, 690, 637; HRMS (ESI-TOF) calcd for $\mathrm{C}_{13} \mathrm{H}_{17} \mathrm{SSi}[\mathrm{M}+\mathrm{H}]^{+}:$233.0820, found: 233.0824 .

4.4.4. tert-Butyldiphenyl((5-(trimethylsilyl)thiophen-2yl)methoxy)silane $11 \mathbf{j}$

The title compound was synthesized from tertbutyldiphenyl(thiophen-2-ylmethoxy)silane according to General Procedure A (yield 89\%). ${ }^{1} \mathrm{H}$ NMR $\left(300 \mathrm{MHz}, \mathrm{CDCl}_{3}\right) \delta 0.33$ (s, 9H), $1.10(\mathrm{~s}, 9 \mathrm{H}), 4.92(\mathrm{~s}, 2 \mathrm{H}), 6.92(\mathrm{~d}, J=3.3 \mathrm{~Hz}, 1 \mathrm{H}), 7.09$ (d, $J=3.3 \mathrm{~Hz}, 1 \mathrm{H}), 7.37-7.44(\mathrm{~m}, 6 \mathrm{H}), 7.71(\mathrm{~d}, J=7.5 \mathrm{~Hz}, 4 \mathrm{H}) ;{ }^{13} \mathrm{C}$ NMR $\left(75 \mathrm{MHz}, \mathrm{CDCl}_{3}\right) \delta-0.01,19.3,26.8,61.5,125.1,127.7$, $129.7,133.3,133.6,135.6,139.3,144.9$; FT-IR (neat, $\mathrm{cm}^{-1}$ ) 3074, 2954, 2857, 1743, 1472, 1427, 1371, 1250, 1111, 995, 836, 704; HRMS (ESI-TOF) calcd for $\mathrm{C}_{24} \mathrm{H}_{32} \mathrm{NaOSSi}_{2}[\mathrm{M}+\mathrm{Na}]^{+}$: 447.1610, found: 447.1606 .

4.4.5. tert-Butyl 2-methyl-5-(trimethylsilyl)-1H-pyrrole-1carboxylate 111

The title compound was synthesized from tert-butyl 2-methyl1H-pyrrole-1-carboxylate according to General Procedure B (yield 52\%). ${ }^{1} \mathrm{H}$ NMR (300 MHz, $\left.\mathrm{CDCl}_{3}\right) \delta 0.25(\mathrm{~s}, 9 \mathrm{H}), 1.60(\mathrm{~s}$, 9H), 2.38 (s, 3H), $5.96(\mathrm{~d}, J=3.0 \mathrm{~Hz}, 1 \mathrm{H}), 6.34(\mathrm{~d}, J=3.0 \mathrm{~Hz}$, $1 \mathrm{H}) ;{ }^{13} \mathrm{C}$ NMR $\left(75 \mathrm{MHz} \mathrm{CDCl}_{3}\right) \delta 0.06,16.6,27.9,83.5,112.9$, 122.1, 134.8, 135.8, 150.8; FT-IR (neat, $\mathrm{cm}^{-1}$ ) 2984, 2901, 1727, 
1570, 1468, 1351, 1239, 1111, 916, 848, 735; HRMS (ESI-TOF) calcd for $\mathrm{C}_{13} \mathrm{H}_{24} \mathrm{NNaO}_{2} \mathrm{Si}[\mathrm{M}+\mathrm{Na}]^{+}: 254.1576$, found: 254.1566 .

4.4.6. tert-Butyl 2-phenyl-5-(trimethylsilyl)-1H-pyrrole-1carboxylate $11 \mathrm{~m}$

The title compound was synthesized from tert-butyl 2-phenyl$1 \mathrm{H}$-pyrrole-1-carboxylate according to General Procedure $\mathrm{B}$ (yield $80 \%)$. ${ }^{1} \mathrm{H}$ NMR $\left(300 \mathrm{MHz}, \mathrm{CDCl}_{3}\right) \delta 0.33(\mathrm{~s}, 9 \mathrm{H}), 1.21$ (s, $9 \mathrm{H}), 6.24(\mathrm{~d}, J=3.3 \mathrm{~Hz}, 1 \mathrm{H}), 6.50(\mathrm{~d}, J=3.3 \mathrm{~Hz}, 1 \mathrm{H}), 7.30-7.37$ $(\mathrm{m}, 5 \mathrm{H}) ;{ }^{13} \mathrm{C}$ NMR $\left(75 \mathrm{MHz}, \mathrm{CDCl}_{3}\right) \delta-0.14,27.2,83.2,114.2$, 122.0, 126.9, 127.6, 128.9, 135.5, 137.1, 138.8, 150.8; FT-IR (neat, $\mathrm{cm}^{-1}$ ) 3063, 2976, 2901, 1739, 1457, 1337, 1242, 1149, 1054, 998, 848, 757, 701; HRMS (ESI-TOF) calcd for $\mathrm{C}_{18} \mathrm{H}_{25} \mathrm{NNaO}_{2} \mathrm{Si}[\mathrm{M}+\mathrm{Na}]^{+}:$338.1552, found: 338.1552 .

4.4.7. tert-Butyl 2-(((tert-butyldiphenylsilyl)oxy)methyl)-5(trimethylsilyl)-1H-pyrrole-1-carboxylate 11n

The title compound was synthesized from tert-butyl 2-(((tertbutyldiphenylsilyl)oxy)methyl)- $1 H$-pyrrole-1-carboxylate

according to General Procedure B (yield 27\%). ${ }^{1} \mathrm{H}$ NMR (300 $\left.\mathrm{MHz} \mathrm{CDCl}_{3}\right) \delta 0.26(\mathrm{~s}, 9 \mathrm{H}), 1.10(\mathrm{~s}, 9 \mathrm{H}), 1.36(\mathrm{~s}, 9 \mathrm{H}), 4.80(\mathrm{~s}$, $2 \mathrm{H}), 6.40(\mathrm{~d}, J=3.3 \mathrm{~Hz}, 1 \mathrm{H}), 6.44(\mathrm{~d}, J=3.3 \mathrm{~Hz}, 1 \mathrm{H}), 7.35-7.42$ $(\mathrm{m}, 6 \mathrm{H}), 7.68(\mathrm{~d}, J=5.4 \mathrm{~Hz}, 4 \mathrm{H}) ;{ }^{13} \mathrm{C}$ NMR $\left(75 \mathrm{MHz} \mathrm{CDCl}_{3}\right) \delta-$ $0.02,19.3,26.8,27.7,62.1,83.8,111.7,122.4,127.7,129.7$, 133.5, 135.6, 136.2, 138.5, 150.2; FT-IR (neat, $\mathrm{cm}^{-1}$ ) 3048, 2958, 2857, 1731, 1476, 1341, 1262, 1167, 1111, 844, 739, 701; HRMS (ESI-TOF) calcd for $\mathrm{C}_{29} \mathrm{H}_{41} \mathrm{NNaO}_{3} \mathrm{Si}_{2}$ [M+Na] 530.2522, found: 530.2518 .

\subsection{8. (4,5-Diphenylfuran-2-yl)trimethylsilane $11 f$}

To a solution of the neutral Au chloride complex $(3.9 \mathrm{mg}, 0.005$ mmol), which can lead to the corresponding cationic complex 19a, and $\operatorname{AgNTf}_{2}(1.9 \mathrm{mg}, 0.005 \mathrm{mmol})$ in toluene $(1.0 \mathrm{~mL})$ was added diol $17(62.0 \mathrm{mg}, 0.20 \mathrm{mmol})$ at room temperature and the reaction mixture was stirred for $3 \mathrm{~h}$. The reaction mixture was directly loaded onto a short silica-gel column (hexane/AcOEt $=$ $1 / 1)$ to remove the catalyst. Purification by silica-gel column chromatography (hexane/AcOEt $=20 / 1)$ gave the product $(85 \%$ yield). ${ }^{1} \mathrm{H}$ NMR $\left(300 \mathrm{MHz}, \mathrm{CDCl}_{3}\right) \delta 0.38(\mathrm{~s}, 9 \mathrm{H}), 6.79(\mathrm{~s}, 1 \mathrm{H})$, 7.30-7.61 (m, 10H); ${ }^{13} \mathrm{C}$ NMR $\left(75 \mathrm{MHz}, \mathrm{CDCl}_{3}\right) \delta-1.6,114.0$, $123.9,126.3,126.9,127.4,128.3,128.5,128.6,131.5,134.7$, 152.4, 159.2; FT-IR (neat, $\mathrm{cm}^{-1}$ ) 3059, 2957, 1604, 1502, 1446, 1269, 1246, 942, 840, 761, 698; HRMS (ESI-TOF) calcd for $\mathrm{C}_{19} \mathrm{H}_{21} \mathrm{OSi}[\mathrm{M}+\mathrm{H}]^{+}:$293.1361, found: 293.1348.

\subsection{General procedure for the catalytic asymmetric Friedel-} Crafts Type reaction with glyoxylate (Table 3)

To a solution of $\mathrm{PdCl}_{2}[(S)$-BINAP $](8.0 \mathrm{mg}, 0.01 \mathrm{mmol})$ in $\mathrm{CH}_{2} \mathrm{Cl}_{2}(2.0 \mathrm{~mL})$ was added $\mathrm{AgSbF}_{6}(7.6 \mathrm{mg}, 0.022 \mathrm{mmol})$ at room temperature under an argon atmosphere. After stirring for $30 \mathrm{~min}$, freshly distilled ethyl glyoxylate $3(61.3 \mathrm{mg}, 0.6 \mathrm{mmol})$ and 2-trimethylsilylated heteroaromatic compound $\mathbf{4 a}$ or $\mathbf{1 1}(0.2$ mmol) were added at $-78-0{ }^{\circ} \mathrm{C}$. The reaction mixture was stirred at $-78-0{ }^{\circ} \mathrm{C}$ for $5-24 \mathrm{~h}$, and then directly loaded onto a silica-gel column (hexane/AcOEt $=1 / 1)$ to remove the catalyst. To a solution of the crude product in THF $(2.0 \mathrm{~mL})$ was added $1 \mathrm{M}$ $\mathrm{HCl}(1.0 \mathrm{~mL})$. After stirring for $1 \mathrm{~h}$ at room temperature, the mixture was extracted with ether. The organic layer was washed with brine, dried over $\mathrm{Na}_{2} \mathrm{SO}_{4}$, and evaporated under reduced pressure. Purification by silica-gel chromatography (hexane/AcOEt $=4 / 1$ ) gave the corresponding alcohol products 6. The enantiomeric excess was determined by chiral HPLC analysis.

\subsection{1. (R)-Ethyl 2-(furan-2-yl)-2-hydroxyacetate $6 \mathrm{a}^{9}$}

${ }^{1} \mathrm{H}$ NMR $\left(300 \mathrm{MHz}, \mathrm{CDCl}_{3}\right) \delta 1.27(\mathrm{t}, J=7.2 \mathrm{~Hz}, 3 \mathrm{H}), 3.33(\mathrm{~d}, J$ $=6.6 \mathrm{~Hz}, 1 \mathrm{H}), 4.25-4.33(\mathrm{~m}, 2 \mathrm{H}), 5.17(\mathrm{~d}, J=5.7 \mathrm{~Hz}, 1 \mathrm{H}), 6.35-$ $6.38(\mathrm{~m}, 2 \mathrm{H}), 7.40(\mathrm{dd}, J=1.8,0.9 \mathrm{~Hz}, 1 \mathrm{H}) ;{ }^{13} \mathrm{C}$ NMR $(75 \mathrm{MHz}$, $\left.\mathrm{CDCl}_{3}\right) \delta 14.0,62.4,66.9,108.5,110.5,142.9,151.0,171.5 ; \mathrm{FT}-$ IR ( $\mathrm{KBr}$ pellet, $\left.\mathrm{cm}^{-1}\right)$ 3422, 2976, 2954, 2359, 1737, 1589, 1495, 1456, 1363, 1292, 1133, 1001, 892, 831; HRMS (APCI-TOF) calcd for $\mathrm{C}_{8} \mathrm{H}_{11} \mathrm{O}_{4}[\mathrm{M}+\mathrm{H}]^{+}: 171.0657$, found: $171.0651 ;[\alpha]_{\mathrm{D}}{ }^{25}=-$ 108.3 ( c $0.80, \mathrm{CHCl}_{3}$ ), 99\% ee; HPLC (column, CHIRALCEL OD-H, Hexane $/ 2$-Propanol $=96 / 4$, flow rate $1.0 \mathrm{~mL} / \mathrm{min}, 20{ }^{\circ} \mathrm{C}$, detection UV $210 \mathrm{~nm}$ ) $t_{\mathrm{R}}$ of minor isomer $11.2 \mathrm{~min}, t_{\mathrm{R}}$ of major isomer $14.1 \mathrm{~min}$. The absolute configuration was determined to be $(R)$ in comparison with the specific rotation of the reported data. $^{9}$

4.5.2. (R)-Ethyl 2-hydroxy-2-(5-methylfuran-2-yl)acetate $6 \mathrm{~b}$ ${ }^{1} \mathrm{H}$ NMR $\left(300 \mathrm{MHz}, \mathrm{CDCl}_{3}\right) \delta 1.27(\mathrm{t}, J=7.2 \mathrm{~Hz}, 3 \mathrm{H}), 2.27(\mathrm{~s}$, $3 \mathrm{H}), 3.32$ (bs, 1H), 4.25-4.33 (m, 2H), $5.11(\mathrm{~s}, 1 \mathrm{H}), 5.93(\mathrm{~d}, J=$ $2.4 \mathrm{~Hz}, 1 \mathrm{H}), 6.24(\mathrm{~d}, J=3.0 \mathrm{~Hz}, 1 \mathrm{H}) ;{ }^{13} \mathrm{C} \mathrm{NMR}(75 \mathrm{MHz}$, $\left.\mathrm{CDCl}_{3}\right) \delta 13.5,14.0,62.4,66.9,106.4,109.6,149.1,152.9$, 171.7; FT-IR (KBr pellet, cm-1) 3466, 2984, 2924, 1743, 1563, 1266, 1216, 1074, 1022, 791; HRMS (ESI-TOF) calcd for $\mathrm{C}_{9} \mathrm{H}_{13} \mathrm{O}_{4}[\mathrm{M}+\mathrm{H}]^{+}:$185.0814, found: $185.0808 ;[\alpha]_{\mathrm{D}}{ }^{25}=-42.3(c$ 1.1, $\mathrm{CHCl}_{3}$ ), 94\% ee; HPLC (column, CHIRALCEL OD-H, Hexane $/ 2$-Propanol $=96 / 4$, flow rate $1.0 \mathrm{ml} / \mathrm{min}, 20^{\circ} \mathrm{C}$, detection UV $210 \mathrm{~nm}$ ) $t_{\mathrm{R}}$ of minor isomer $12.5 \mathrm{~min}, t_{\mathrm{R}}$ of major isomer 14.7 min. The absolute configuration was tentatively assigned by analogy of the specific rotation.

\subsection{3. (R)-Ethyl 2-hydroxy-2-(5-phenylfuran-2-yl)acetate $6 \mathrm{c}$} ${ }^{1} \mathrm{H}$ NMR (300 MHz, $\left.\mathrm{CDCl}_{3}\right) \delta 1.29(\mathrm{t}, J=7.2 \mathrm{~Hz}, 3 \mathrm{H}), 3.41(\mathrm{~d}, J$ $=7.8 \mathrm{~Hz}, 1 \mathrm{H}), 4.26-4.35(\mathrm{~m}, 2 \mathrm{H}), 5.22(\mathrm{~d}, J=7.8 \mathrm{~Hz}, 1 \mathrm{H}), 6.45$ $(\mathrm{d}, J=3.3 \mathrm{~Hz}, 1 \mathrm{H}), 6.61(\mathrm{~d}, J=3.3 \mathrm{~Hz}, 1 \mathrm{H}), 7.24-7.40(\mathrm{~m}, 3 \mathrm{H})$, $7.65(\mathrm{~d}, J=5.4 \mathrm{~Hz}, 2 \mathrm{H}) ;{ }^{13} \mathrm{C} \mathrm{NMR}(75 \mathrm{MHz}, \mathrm{CDCl} 3) \delta 14.0$, 62.6, 67.0, 105.7, 110.7, 123.9, 127.7, 128.7, 130.4, 150.4, 154.4, 171.5; FT-IR (KBr pellet, cm-1) 3462, 2981, 2927, 1739, 1484, 1448, 1263, 1211, 1074, 1023, 758, 695; HRMS (ESI-TOF) calcd for $\mathrm{C}_{14} \mathrm{H}_{14} \mathrm{NaO}_{4}[\mathrm{M}+\mathrm{Na}]^{+}$: 269.0790, found: 269.0803; $[\alpha]_{\mathrm{D}}^{25}=-44.4\left(c 0.86, \mathrm{CHCl}_{3}\right), 97 \% e e ; \mathrm{HPLC}$ (column, CHIRALCEL OD-H, Hexane/2-Propanol $=96 / 4$, flow rate 1.0 $\mathrm{mL} / \mathrm{min}, 20{ }^{\circ} \mathrm{C}$, detection UV $210 \mathrm{~nm}$ ) $t_{\mathrm{R}}$ of minor isomer 15.9 $\min , t_{\mathrm{R}}$ of major isomer $22.4 \mathrm{~min}$. The absolute configuration was tentatively assigned by analogy of the specific rotation.

4.5.4. (R)-Ethyl 2-(5-(( (tertbutyldiphenylsilyl)oxy)methyl)furan-2-yl)-2-hydroxyacetate 6d

${ }^{1} \mathrm{H}$ NMR (300 MHz, CDCl3) $\delta 1.05(\mathrm{~s}, 9 \mathrm{H}), 1.26(\mathrm{t}, J=7.2 \mathrm{~Hz}$, $3 \mathrm{H}), 3.30$ (bs, 1H), 4.21-4.33 (m, 2H), 4.62 (s, 1H), 5.14 (s, 2H), $6.12(\mathrm{~d}, J=3.3 \mathrm{~Hz}, 1 \mathrm{H}), 6.29(\mathrm{~d}, J=3.3 \mathrm{~Hz}, 1 \mathrm{H}), 7.38-7.70(\mathrm{~m}$, $10 \mathrm{H}) ;{ }^{13} \mathrm{C}$ NMR $(75 \mathrm{MHz}, \mathrm{CDCl} 3) \delta 14.1,19.2,26.7,58.9,62.5$, 67.0, 108.2, 109.3, 127.6, 129.7, 133.2, 135.6, 150.3, 154.6, 171.5; FT-IR (KBr pellet, cm-1) 3488, 2959, 2931, 2857, 1739, 1428, 1219, 1110, 1073, 1015, 823, 701, 610; HRMS (ESI-TOF) calcd for $\mathrm{C}_{25} \mathrm{H}_{30} \mathrm{NaO}_{5} \mathrm{Si}[\mathrm{M}+\mathrm{Na}]^{+}$: 461.1760, found: 461.1772; $[\alpha]_{\mathrm{D}}^{25}-35.6\left(c 1.1, \mathrm{CHCl}_{3}\right), 99 \% e e ;$ HPLC (column, CHIRALCEL OD-H, Hexane/2-Propanol = 96/4, flow rate 1.0 $\mathrm{mL} / \mathrm{min}, 20{ }^{\circ} \mathrm{C}$, detection UV $210 \mathrm{~nm}$ ) $t_{\mathrm{R}}$ of minor isomer 17.2 $\mathrm{min}, t_{\mathrm{R}}$ of major isomer $20.6 \mathrm{~min}$. The absolute configuration was tentatively assigned by analogy of the specific rotation.

\subsection{5. (R)-Ethyl 2-(3,5-diphenylfuran-2-yl)-2-hydroxyacetate} $6 \mathrm{e}$

${ }^{1} \mathrm{H}$ NMR (300 MHz, $\left.\mathrm{CDCl}_{3}\right) \delta 1.25(\mathrm{t}, J=7.2 \mathrm{~Hz}, 3 \mathrm{H}), 3.60(\mathrm{~d}, J$ $=6.0 \mathrm{~Hz}, 1 \mathrm{H}), 4.25-4.33(\mathrm{~m}, 2 \mathrm{H}), 5.35(\mathrm{~d}, J=6.0 \mathrm{~Hz}, 1 \mathrm{H}), 6.83$ (s, 1H), 7.28-7.49 (m, 6H), $7.58(\mathrm{~d}, J=2.4 \mathrm{~Hz}, 2 \mathrm{H}), 7.59(\mathrm{~d}, J=$ $2.4 \mathrm{~Hz}, 2 \mathrm{H}) ;{ }^{13} \mathrm{C}$ NMR $\left(75 \mathrm{MHz}, \mathrm{CDCl}_{3}\right) \delta 14.0,62.6,65.5$, 
106.8, 124.0, 127.6, 127.89, 127.94, 128.2, 128.7, 128.8, 130.1, 132.5, 145.5, 153.5, 171.6; FT-IR (KBr pellet, $\mathrm{cm}^{-1}$ ) 3481, 3059, 2980, 1739, 1486, 1451, 1264, 1222, 1071, 758, 696; HRMS (ESI-TOF) calcd for $\mathrm{C}_{20} \mathrm{H}_{18} \mathrm{KO}_{4}[\mathrm{M}+\mathrm{K}]^{+}:$361.0842, found: $361.0830 ;[\alpha]_{\mathrm{D}}^{25}=-19.5\left(c 1.05, \mathrm{CHCl}_{3}\right), 82 \%$ ee; HPLC (column, CHIRALCEL OD-H, Hexane/2-Propanol = 96/4, flow rate $1.0 \mathrm{~mL} / \mathrm{min}, 20^{\circ} \mathrm{C}$, detection UV $210 \mathrm{~nm}$ ) $t_{\mathrm{R}}$ of major isomer $25.8 \mathrm{~min}, t_{\mathrm{R}}$ of minor isomer $37.6 \mathrm{~min}$. The absolute configuration was tentatively assigned by analogy of the specific rotation.

\subsection{6. (R)-Ethyl 2-(4,5-diphenylfuran-2-yl)-2-hydroxyacetate 6f}

${ }^{1} \mathrm{H}$ NMR $\left(300 \mathrm{MHz}, \mathrm{CDCl}_{3}\right) \delta 1.32(\mathrm{t}, J=7.2 \mathrm{~Hz}, 3 \mathrm{H}), 3.40(\mathrm{bs}$, $1 \mathrm{H}), 4.27-4.40(\mathrm{~m}, 2 \mathrm{H}), 5.25(\mathrm{~s}, 1 \mathrm{H}), 6.53(\mathrm{~s}, 1 \mathrm{H}), 7.30-7.52(\mathrm{~m}$, $10 \mathrm{H}) ;{ }^{13} \mathrm{C} \mathrm{NMR}\left(75 \mathrm{MHz}, \mathrm{CDCl}_{3}\right) \delta 14.1,62.7,67.0,112.8$, 123.0, 126.3, 127.3, 127.8, 128.4, 128.63, 128.64, 130.7, 133.9, 148.9, 149.8, 171.4; FT-IR (KBr pellet, $\mathrm{cm}^{-1}$ ) 3390, 2920, 2853, 1743, 1671, 1446, 1252, 1188, 764, 697; HRMS (ESI-TOF) calcd for $\mathrm{C}_{20} \mathrm{H}_{18} \mathrm{NaO}_{4}[\mathrm{M}+\mathrm{Na}]^{+}$: 345.1103, found: 345.1116; $[\alpha]_{\mathrm{D}}^{25}=-36.1\left(c 0.88, \mathrm{CHCl}_{3}\right), 99 \% e e ;$ HPLC (column, CHIRALCEL OD-H, Hexane/2-Propanol $=96 / 4$, flow rate 1.0 $\mathrm{mL} / \mathrm{min}, 20{ }^{\circ} \mathrm{C}$, detection UV $210 \mathrm{~nm}$ ) $t_{\mathrm{R}}$ of minor isomer 18.2 $\min , t_{\mathrm{R}}$ of major isomer $26.2 \mathrm{~min}$. The absolute configuration was tentatively assigned by analogy of the specific rotation.

\subsection{7. (R)-Ethyl 2-hydroxy-2-(thiophen-2-yl)acetate $6 \mathrm{~g}^{\mathbf{9}}$}

${ }^{1} \mathrm{H} \mathrm{NMR}\left(300 \mathrm{MHz}, \mathrm{CDCl}_{3}\right) \delta 1.29(\mathrm{t}, J=7.2 \mathrm{~Hz}, 3 \mathrm{H}), 3.54(\mathrm{~d}, J$ $=6.3 \mathrm{~Hz}, 1 \mathrm{H}), 4.21-4.36(\mathrm{~m}, 2 \mathrm{H}), 5.40(\mathrm{~d}, J=6.3 \mathrm{~Hz}, 1 \mathrm{H}), 6.99$ $(\mathrm{dd}, J=5.1,3.6 \mathrm{~Hz} 1 \mathrm{H}), 7.10(\mathrm{~d}, J=3.6 \mathrm{~Hz}, 1 \mathrm{H}), 7.28(\mathrm{dd}, J=$ $5.1,1.2 \mathrm{~Hz} 1 \mathrm{H}) ;{ }^{13} \mathrm{C} \mathrm{NMR}\left(75 \mathrm{MHz}, \mathrm{CDCl}_{3}\right) \delta 14.0,62.6,69.1$, 125.3, 125.6, 126.9, 141.5, 172.6; FT-IR (neat, $\mathrm{cm}^{-1}$ ) 3453, 3012, 2961, 2912, 1751, 1685, 1512, 1399, 1328, 1249, 1188, 1090, 1032, 918, 873; HRMS (APCI-TOF) calcd for $\mathrm{C}_{8} \mathrm{H}_{10} \mathrm{NaO}_{3} \mathrm{~S}$ $[\mathrm{M}+\mathrm{Na}]^{+}: 209.0248$, found: $209.0250 ;[\alpha]_{\mathrm{D}}{ }^{25}=-73.8(c 0.55$, $\mathrm{CHCl}_{3}$ ), 98\% ee; HPLC (column, CHIRALPAK AD-H, Hexane/2-Propanol $=96 / 4$, flow rate $1.0 \mathrm{~mL} / \mathrm{min}, 20{ }^{\circ} \mathrm{C}$, detection UV $210 \mathrm{~nm}$ ) $t_{\mathrm{R}}$ of minor isomer $15.8 \mathrm{~min}, t_{\mathrm{R}}$ of major isomer $17.7 \mathrm{~min}$. The absolute configuration was tentatively assigned by analogy of the specific rotation.

\subsection{8. (R)-Ethyl 2-hydroxy-2-(5-methylthiophen-2-yl)acetate} $6 h$

${ }^{1} \mathrm{H}$ NMR (300 MHz, $\left.\mathrm{CDCl}_{3}\right) \delta 1.29(\mathrm{t}, J=7.2 \mathrm{~Hz}, 3 \mathrm{H}), 2.45$ (s, $3 \mathrm{H}), 3.42(\mathrm{~d}, J=6.6 \mathrm{~Hz}, 1 \mathrm{H}), 4.21-4.35(\mathrm{~m}, 2 \mathrm{H}), 5.30(\mathrm{~d}, J=6.6$ $\mathrm{Hz}, 1 \mathrm{H}), 6.62(\mathrm{~d}, J=3.6 \mathrm{~Hz}, 1 \mathrm{H}), 6.87(\mathrm{~d}, J=3.6 \mathrm{~Hz}, 1 \mathrm{H}) ;{ }^{13} \mathrm{C}$ NMR $\left(75 \mathrm{MHz}, \mathrm{CDCl}_{3}\right) \delta 14.0,15.3,62.4,69.1,124.9,125.4$, 138.8, 140.5, 172.6; FT-IR (neat, $\mathrm{cm}^{-1}$ ) 3480, 2982, 2923, 1735, 1448, 1367, 1267, 1213, 1072, 1022, 799; HRMS (ESI-TOF) calcd for $\mathrm{C}_{9} \mathrm{H}_{12} \mathrm{NaO}_{3} \mathrm{~S}[\mathrm{M}+\mathrm{Na}]^{+}$: 223.0405, found: 223.0394; $[\alpha]_{\mathrm{D}}^{25}=-59.7\left(c 1.16, \mathrm{CHCl}_{3}\right), 99 \%$ ee; HPLC (column, CHIRALPAK AD-3, Hexane/2-Propanol $=97 / 3$, flow rate 0.6 $\mathrm{mL} / \mathrm{min}, 20{ }^{\circ} \mathrm{C}$, detection UV $210 \mathrm{~nm}$ ) $t_{\mathrm{R}}$ of minor isomer 35.2 $\min , t_{\mathrm{R}}$ of major isomer $39.6 \mathrm{~min}$. The absolute configuration was tentatively assigned by analogy of the specific rotation.

\subsection{9. (R)-Ethyl 2-hydroxy-2-(5-phenylthiophen-2-yl)acetate} $6 \mathbf{i}$

${ }^{1} \mathrm{H}$ NMR $\left(300 \mathrm{MHz}, \mathrm{CDCl}_{3}\right) \delta 1.33(\mathrm{t}, J=7.2 \mathrm{~Hz}, 3 \mathrm{H}), 3.58(\mathrm{~d}, J$ $=6.0 \mathrm{~Hz}, 1 \mathrm{H}), 4.30-4.35(\mathrm{~m}, 2 \mathrm{H}), 5.41(\mathrm{~d}, J=4.8 \mathrm{~Hz}, 1 \mathrm{H}), 7.08$ $(\mathrm{d}, J=3.9 \mathrm{~Hz}, 1 \mathrm{H}), 7.20(\mathrm{~d}, J=3.6 \mathrm{~Hz}, 1 \mathrm{H}), 7.28-7.42(\mathrm{~m}, 3 \mathrm{H})$, $7.40(\mathrm{dd}, J=7.2,1.5 \mathrm{~Hz}, 2 \mathrm{H}) ;{ }^{13} \mathrm{C} \mathrm{NMR}\left(75 \mathrm{MHz}, \mathrm{CDCl}_{3}\right) \delta$ 14.0, 62.7, 69.2, 122.8, 125.7, 126.3, 127.7, 128.9, 134.1, 140.6, 144.7, 172.6; FT-IR (KBr pellet, $\mathrm{cm}^{-1}$ ) 3454, 2980, 2932, 1727, 1460, 1382, 1213, 1196, 1083, 1022, 751, 685; HRMS (ESITOF) calcd for $\mathrm{C}_{14} \mathrm{H}_{14} \mathrm{NaO}_{3} \mathrm{~S} \quad[\mathrm{M}+\mathrm{Na}]^{+}:$285.0561, found:
285.0573; $[\alpha]_{\mathrm{D}}{ }^{25}=-13.6\left(c 1.00, \mathrm{CHCl}_{3}\right), 99 \% e e ;$ HPLC (column, CHIRALCEL OD-H, Hexane/2-Propanol = 96/4, flow rate $1.0 \mathrm{~mL} / \mathrm{min}, 20{ }^{\circ} \mathrm{C}$, detection UV $\left.210 \mathrm{~nm}\right) t_{\mathrm{R}}$ of minor isomer $18.1 \mathrm{~min}, t_{\mathrm{R}}$ of major isomer $22.0 \mathrm{~min}$. The absolute configuration was tentatively assigned by analogy of the specific rotation.

4.5.10. (R)-Ethyl 2-(5-(( tertbutyldiphenylsilyl)oxy)methyl)thiophen-2-yl)-2hydroxyacetate $\mathbf{6 j}$

${ }^{1} \mathrm{H}$ NMR $\left(300 \mathrm{MHz}, \mathrm{CDCl}_{3}\right) \delta 1.08(\mathrm{~s}, 9 \mathrm{H}), 1.31(\mathrm{t}, J=7.2 \mathrm{~Hz}$, $3 \mathrm{H}), 3.46(\mathrm{~d}, J=6.6 \mathrm{~Hz}, 1 \mathrm{H}), 4.26-4.34(\mathrm{~m}, 2 \mathrm{H}), 4.83(\mathrm{~s}, 2 \mathrm{H})$, $5.36(\mathrm{~d}, J=6.3 \mathrm{~Hz}, 1 \mathrm{H}), 6.70(\mathrm{~d}, J=3.6 \mathrm{~Hz}, 1 \mathrm{H}), 6.92(\mathrm{~d}, J=3.6$ $\mathrm{Hz}, 1 \mathrm{H}), 7.37-7.44(\mathrm{~m}, 6 \mathrm{H}), 7.69(\mathrm{~d}, J=7.8 \mathrm{~Hz}, 4 \mathrm{H}) ;{ }^{13} \mathrm{C} \mathrm{NMR}$ $\left(75 \mathrm{MHz}, \mathrm{CDCl}_{3}\right) \delta 14.0,19.2,26.7,61.6,62.5,69.2,123.3$, $124.9,127.7,129.7,133.1,135.6,140.3,145.4,172.5$; FT-IR $\left(\mathrm{KBr}\right.$ pellet, $\left.\mathrm{cm}^{-1}\right)$ 3487, 3068, 2934, 2856, 1739, 1428, 1268, 1211, 1107, 1076, 826, 705; HRMS (ESI-TOF) calcd for $\mathrm{C}_{25} \mathrm{H}_{30} \mathrm{NaO}_{4} \mathrm{SSi}[\mathrm{M}+\mathrm{Na}]^{+}: 477.1532$, found: $477.1544 ;[\alpha]_{\mathrm{D}}{ }^{25}=-$ 24.5 (c 1.12, $\mathrm{CHCl}_{3}$ ), 99\% ee; HPLC (column, CHIRALCEL OD-H, Hexane $/ 2$-Propanol $=96 / 4$, flow rate $1.0 \mathrm{~mL} / \mathrm{min}, 20{ }^{\circ} \mathrm{C}$, detection UV $210 \mathrm{~nm}) t_{\mathrm{R}}$ of minor isomer $19.5 \mathrm{~min}, t_{\mathrm{R}}$ of major isomer $43.2 \mathrm{~min}$. The absolute configuration was tentatively assigned by analogy of the specific rotation.

4.5.11. (R)-tert-Butyl 2-(2-ethoxy-1-hydroxy-2-oxoethyl)-1Hpyrrole-1-carboxylate $6 \mathrm{k}^{\mathbf{9}}$

${ }^{1} \mathrm{H}$ NMR (300 MHz, $\left.\mathrm{CDCl}_{3}\right) \delta 1.24(\mathrm{t}, J=7.2 \mathrm{~Hz}, 3 \mathrm{H}), 1.57(\mathrm{~s}$, $9 \mathrm{H}), 4.04(\mathrm{~d}, J=8.1 \mathrm{~Hz}, 1 \mathrm{H}), 4.18-4.26(\mathrm{~m}, 2 \mathrm{H}), 5.37$ (d, $J=7.5$ $\mathrm{Hz}, 1 \mathrm{H}), 6.12(\mathrm{dd}, J=3.6,3.3 \mathrm{~Hz}, 1 \mathrm{H}), 6.25(\mathrm{dd}, J=3.3,1.5 \mathrm{~Hz}$, $1 \mathrm{H}), 7.38(\mathrm{dd}, J=3.6,1.5 \mathrm{~Hz}, 1 \mathrm{H}) ;{ }^{13} \mathrm{C} \mathrm{NMR}\left(75 \mathrm{MHz}, \mathrm{CDCl}_{3}\right) \delta$ $14.2,27.9,61.6,67.8,84.8,110.4,115.5,122.5,131.7,149.8$, 171.6; FT-IR (neat, $\mathrm{cm}^{-1}$ ) 3491, 3154, 2982, 2938, 1739, 1478, $1423,1395,1350,1240,1145,1053,950,847$; HRMS (ESITOF) calcd for $\mathrm{C}_{13} \mathrm{H}_{19} \mathrm{NNaO}_{5}[\mathrm{M}+\mathrm{Na}]^{+}$: 292.1161, found: 292.1160; $[\alpha]_{\mathrm{D}}{ }^{25}=-50.7\left(c 0.96, \mathrm{CHCl}_{3}\right), 99 \% e e ;$ HPLC (column, CHIRALPAK AD-H, Hexane/2-Propanol = 97/3, flow rate $1.0 \mathrm{~mL} / \mathrm{min}, 20^{\circ} \mathrm{C}$, detection UV $\left.254 \mathrm{~nm}\right) t_{\mathrm{R}}$ of minor isomer $18.0 \mathrm{~min}, t_{\mathrm{R}}$ of major isomer $22.6 \mathrm{~min}$. The absolute configuration was tentatively assigned by analogy of the specific rotation.

4.5.12. (R)-tert-Butyl 2-(2-ethoxy-1-hydroxy-2-oxoethyl)-5methyl-1H-pyrrole-1-carboxylate 61

${ }^{1} \mathrm{H}$ NMR $\left(300 \mathrm{MHz}, \mathrm{CDCl}_{3}\right) \delta 1.26(\mathrm{t}, J=7.2 \mathrm{~Hz}, 3 \mathrm{H}), 1.58(\mathrm{~s}$, 9H), $4.05(\mathrm{~d}, J=9.3 \mathrm{~Hz}, 1 \mathrm{H}), 4.18-4.25(\mathrm{~m}, 2 \mathrm{H}), 5.27$ (d, $J=9.3$ $\mathrm{Hz}, 1 \mathrm{H}), 5.87(\mathrm{~d}, J=3.3 \mathrm{~Hz}, 1 \mathrm{H}), 6.12(\mathrm{dd}, J=3.3 \mathrm{~Hz}, 1 \mathrm{H}) ;{ }^{13} \mathrm{C}$ NMR $\left(75 \mathrm{MHz}, \mathrm{CDCl}_{3}\right) \delta 14.2,16.7,28.0,61.6,68.6,85.0$, 110.1, 114.3, 131.8, 132.9, 151.0, 171.9; FT-IR (neat, $\mathrm{cm}^{-1}$ ) 3504, 2978, 2931, 1739, 1392, 1347, 1259, 1129, 1071, 850, 788; HRMS (ESI-TOF) calcd for $\mathrm{C}_{14} \mathrm{H}_{21} \mathrm{NNaO}_{5}[\mathrm{M}+\mathrm{Na}]^{+}$: 306.1317 , found: $306.1317 ;[\alpha]_{\mathrm{D}}{ }^{25}=-40.3\left(c 0.86, \mathrm{CHCl}_{3}\right), 98 \%$ ee; HPLC (column, CHIRALPAK AD-H, Hexane/2-Propanol = 97/3, flow rate $1.0 \mathrm{~mL} / \mathrm{min}, 20^{\circ} \mathrm{C}$, detection UV $210 \mathrm{~nm}$ ) $t_{\mathrm{R}}$ of minor isomer $13.5 \mathrm{~min}, t_{\mathrm{R}}$ of major isomer $15.5 \mathrm{~min}$. The absolute configuration was tentatively assigned by analogy of the specific rotation.

4.5.13. (R)-tert-Butyl 2-(2-ethoxy-1-hydroxy-2-oxoethyl)-5phenyl-1H-pyrrole-1-carboxylate $6 \mathrm{~m}$

${ }^{1} \mathrm{H}$ NMR $\left(300 \mathrm{MHz}, \mathrm{CDCl}_{3}\right) \delta 1.17(\mathrm{~s}, 9 \mathrm{H}), 1.29(\mathrm{t}, J=7.2 \mathrm{~Hz}$, $3 \mathrm{H}), 4.17-4.30(\mathrm{~m}, 3 \mathrm{H}), 5.37(\mathrm{~d}, J=9.0 \mathrm{~Hz}, 1 \mathrm{H}), 6.12(\mathrm{~d}, J=3.3$ $\mathrm{Hz}, 1 \mathrm{H}), 6.27$ (d, $J=3.3 \mathrm{~Hz}, 1 \mathrm{H}), 7.27-7.35(\mathrm{~m}, 5 \mathrm{H}) ;{ }^{13} \mathrm{C} \mathrm{NMR}$ $\left(75 \mathrm{MHz}, \mathrm{CDCl}_{3}\right) \delta 14.2,27.1,61.7,68.4,84.8,112.6,113.9$, $127.2,127.7,128.8,133.0,134.8,136.7,150.9,171.7$; FT-IR (neat, $\mathrm{cm}^{-1}$ ) 3488, 2979, 2928, 1738, 1370, 1313, 1145, 848, 758, 
700; HRMS (ESI-TOF) calcd for $\mathrm{C}_{19} \mathrm{H}_{23} \mathrm{KNO}_{5}[\mathrm{M}+\mathrm{K}]^{+}$: 384.1213, found: $384.1214 ;[\alpha]_{\mathrm{D}}{ }^{25}=-15.2\left(c 0.64, \mathrm{CHCl}_{3}\right), 96 \%$ $e e$; HPLC (column, CHIRALPAK AD-H, Hexane/2-Propanol = $97 / 3$, flow rate $1.0 \mathrm{~mL} / \mathrm{min}, 20^{\circ} \mathrm{C}$, detection UV $\left.210 \mathrm{~nm}\right) t_{\mathrm{R}}$ of minor isomer $15.1 \mathrm{~min}, t_{\mathrm{R}}$ of major isomer $17.4 \mathrm{~min}$. The absolute configuration was tentatively assigned by analogy of the specific rotation.

4.5.14. (R)-tert-Butyl 2-((tert-butyldiphenylsilyl)oxy)methyl)5(2-ethoxy-1-hydroxy-2-oxoethyl)-1H-pyrrole-1-carboxylate (6n)

${ }^{1} \mathrm{H}$ NMR $\left(300 \mathrm{MHz}, \mathrm{CDCl}_{3}\right) \delta 1.09(\mathrm{~s}, 9 \mathrm{H}), 1.27(\mathrm{t}, J=7.2 \mathrm{~Hz}$, $3 \mathrm{H}), 4.03(\mathrm{~d}, J=9.0 \mathrm{~Hz}, 1 \mathrm{H}), 4.22(\mathrm{q}, J=7.2 \mathrm{~Hz}, 2 \mathrm{H}), 4.75(\mathrm{~s}$, $2 \mathrm{H}), 5.31(\mathrm{~d}, J=9.0 \mathrm{~Hz}, 1 \mathrm{H}), 6.22(\mathrm{~d}, J=3.3 \mathrm{~Hz}, 1 \mathrm{H}), 6.28(\mathrm{~d}, J$ $=3.3 \mathrm{~Hz}, 1 \mathrm{H}), 7.35-7.43(\mathrm{~m}, 6 \mathrm{H}), 7.64-7.68(\mathrm{~m}, 4 \mathrm{H}) ;{ }^{13} \mathrm{C} \mathrm{NMR}$ $\left(75 \mathrm{MHz}, \mathrm{CDCl}_{3}\right) \delta 14.2,19.3,26.8,27.6,61.6,61.8,68.5,85.3$, $110.2,114.7,127.7,129.7132 .4,133.3,135.5,136.3,150.4$, 171.7; FT-IR (neat, $\mathrm{cm}^{-1}$ ) 3530, 3070, 2931, 2857, 1736, 1427, 1371, 1335, 1256, 1111, 704; HRMS (ESI-TOF) calcd for $\mathrm{C}_{30} \mathrm{H}_{39} \mathrm{NNaO}_{5} \mathrm{Si}[\mathrm{M}+\mathrm{Na}]^{+}: 560.2444$, found: $560.2437 ;[\alpha]_{\mathrm{D}}{ }^{25}-$ 25.6 (c 1.03, $\mathrm{CHCl}_{3}$ ), 99\% ee; HPLC (column, CHIRALPAK AD-H, Hexane/2-Propanol $=97 / 3$, flow rate $1.0 \mathrm{~mL} / \mathrm{min}, 20^{\circ} \mathrm{C}$, detection UV $210 \mathrm{~nm}$ ) $t_{\mathrm{R}}$ of minor isomer $12.4 \mathrm{~min}, t_{\mathrm{R}}$ of major isomer $14.9 \mathrm{~min}$. The absolute configuration was tentatively assigned by analogy of the specific rotation.

\subsection{Synthesis of pyranone (Scheme 4)}

To a solution of $\mathbf{6 a}(851 \mathrm{mg}, 5.0 \mathrm{mmol}, 99 \%$ ee $)$ in THF $(30 \mathrm{~mL})$ and $\mathrm{MeOH}(5.0 \mathrm{~mL})$ at $0{ }^{\circ} \mathrm{C}$ was added $\mathrm{NaBH}_{4}(605 \mathrm{mg}, 16$ mmol) under an argon atmosphere, and the reaction mixture was warmed up to room temperature. After stirring for $12 \mathrm{~h}$, saturated aqueous $\mathrm{NH}_{4} \mathrm{Cl}$ was added. The organic layer was separated, and the aqueous layer was extracted with ether. The combined organic layer was washed with brine, dried over $\mathrm{Na}_{2} \mathrm{SO}_{4}$, and evaporated under reduced pressure. Purification by silica-gel chromatography (hexane/AcOEt $=1 / 1)$ gave the diol $12(90 \%$ yield). ${ }^{1} \mathrm{H}$ NMR $\left(300 \mathrm{MHz}, \mathrm{CDCl}_{3}\right) \delta 3.54(\mathrm{br}, 1 \mathrm{H}), 3.76-3.80(\mathrm{br}$ s, $2 \mathrm{H}), 3.95(\mathrm{br}, 1 \mathrm{H}), 4.75(\mathrm{~d}, J=5.9 \mathrm{~Hz}, 1 \mathrm{H}), 6.23(\mathrm{dd}, J=4.0$, $0.6 \mathrm{~Hz}, 1 \mathrm{H}), 6.27$ (dd, $J=4.0,1.8 \mathrm{~Hz}, 1 \mathrm{H}), 7.30$ (dd, $J=1.8,0.6$ $\mathrm{Hz}, 1 \mathrm{H}) ;{ }^{13} \mathrm{C}$ NMR $\left(75 \mathrm{MHz}, \mathrm{CDCl}_{3}\right) \delta 65.0,68.3,106.9,110.3$, 142.2, 153.6 .

To a solution of diol $12(256 \mathrm{mg}, 2.0 \mathrm{mmol})$ in $\mathrm{CH}_{2} \mathrm{Cl}_{2}(10 \mathrm{~mL})$ were added triethylamine $(554 \mu \mathrm{L}, 4.0 \mathrm{mmol})$ and pivaloyl chloride $(270 \mu \mathrm{L}, 2.2 \mathrm{mmol})$ at $-78{ }^{\circ} \mathrm{C}$ under an argon atmosphere. After the reaction mixture was allowed to slowly warm to $0{ }^{\circ} \mathrm{C}$ and stirred for $5 \mathrm{~h}$, saturated aqueous $\mathrm{NaHCO}_{3}$ was added. The organic layer was separated, and the aqueous layer was extracted with ether. The combined organic layer was washed with brine, dried over $\mathrm{Na}_{2} \mathrm{SO}_{4}$, and evaporated under reduced pressure. Purification by silica-gel chromatography (hexane/AcOEt $=5 / 1)$ gave the piv-protected product $13(87 \%$ yield). ${ }^{1} \mathrm{H}$ NMR $\left(300 \mathrm{MHz}, \mathrm{CDCl}_{3}\right) \delta 1.14(\mathrm{~s}, 9 \mathrm{H}), 3.07$ (br, $\left.1 \mathrm{H}\right)$, $4.30(\mathrm{dd}, J=5.7,4.8 \mathrm{~Hz}, 1 \mathrm{H}), 4.33(\mathrm{dd}, J=5.7,4.8 \mathrm{~Hz}, 1 \mathrm{H})$, $4.90(\mathrm{dd}, J=5.7,5.7 \mathrm{~Hz}, 1 \mathrm{H}), 6.26-6.32(\mathrm{~m}, 2 \mathrm{H}), 7.34(\mathrm{dd}, J=$ $1.8,0.9 \mathrm{~Hz}, 1 \mathrm{H}) ;{ }^{13} \mathrm{C}$ NMR $\left(75 \mathrm{MHz}, \mathrm{CDCl}_{3}\right) \delta 27.0,38.8,66.2$, 66.3, 107.1, 110.2, 142.3, 153.2, 178.6.

An Achmatowicz rearrangement was attempted in this step according to O'Doherty's procedure. ${ }^{13 \mathrm{~b}}$ To a solution of $\mathbf{1 3}$ (212 $\mathrm{mg}, 1.0 \mathrm{mmol}), \mathrm{NaOAc} \cdot 3 \mathrm{H}_{2} \mathrm{O}(149 \mathrm{mg}, 1.1 \mathrm{mmol})$, and $\mathrm{NaHCO}_{3}$ $(167 \mathrm{mg} 2.0 \mathrm{mmol})$ in THF $(2.0 \mathrm{~mL})$ and $\mathrm{H}_{2} \mathrm{O}(1.0 \mathrm{~mL})$ was added NBS (195 mg, $1.1 \mathrm{mmol}$ ) at $0{ }^{\circ} \mathrm{C}$. After stirring for $1 \mathrm{~h}$, the mixture was quenched by saturated aqueous $\mathrm{NaHCO}_{3}$ and extracted with ether. The organic layer was washed with brine, dried over $\mathrm{Na}_{2} \mathrm{SO}_{4}$, and evaporated under reduced pressure. Crude 14 was obtained and used for the next step without further purification. trans-14: ${ }^{1} \mathrm{H}$ NMR $\left(300 \mathrm{MHz}, \mathrm{CDCl}_{3}\right) \delta 1.19$ (s,
9H), 4.41-4.49 (m, 2H), 4.73 (dd, $J=4.2,4.2 \mathrm{~Hz}, 1 \mathrm{H}), 5.61$ (d, $J$ $=7.5 \mathrm{~Hz}, 1 \mathrm{H}), 6.08(\mathrm{~d}, J=10.9 \mathrm{~Hz}, 1 \mathrm{H}), 6.91(\mathrm{dd}, J=10.9,7.5$ $\mathrm{Hz}, 1 \mathrm{H}$ ). To a solution of $\mathbf{1 4}$ (without purification) and DMAP (cat.) in $\mathrm{CH}_{2} \mathrm{Cl}_{2}(5.0 \mathrm{~mL})$ were added triethylamine $(277 \mu \mathrm{L}, 2.0$ mmol) and benzoyl chloride $(127 \mu \mathrm{L}, 1.1 \mathrm{mmol})$ at $-78^{\circ} \mathrm{C}$ under an argon atmosphere. After stirring for $1 \mathrm{~h}$ at $-40{ }^{\circ} \mathrm{C}$, saturated aqueous $\mathrm{NaHCO}_{3}$ was added. The organic layer was separated, and the aqueous layer was extracted with ether. The combined organic layer was washed with brine, dried over $\mathrm{Na}_{2} \mathrm{SO}_{4}$, and evaporated under reduced pressure. Purification by silica-gel chromatography (hexane/AcOEt $=5 / 1$ ) gave pyranone $\mathbf{1 5}^{\mathbf{1 3 b}}$ (86\% yield, trans:cis 4:1, 2 steps). trans-15: ${ }^{1} \mathrm{H}$ NMR $(300 \mathrm{MHz}$, $\left.\mathrm{CDCl}_{3}\right) \delta 1.19(\mathrm{~s}, 9 \mathrm{H}), 4.50-4.55(\mathrm{~m}, 2 \mathrm{H}), 4.82(\mathrm{dd}, J=5.7,5.7$ $\mathrm{Hz}, 1 \mathrm{H}), 6.31$ (d, $J=10.5 \mathrm{~Hz}, 1 \mathrm{H}), 6.83$ (d, $J=6.6 \mathrm{~Hz}, 1 \mathrm{H}), 7.02$ $(\mathrm{dd}, J=10.5,6.6 \mathrm{~Hz}, 1 \mathrm{H}), 7.41-7.50(\mathrm{~m}, 2 \mathrm{H}), 7.61-7.67(\mathrm{~m}, 1 \mathrm{H})$, $8.01(\mathrm{dd}, J=8.7,1.5 \mathrm{~Hz}, 2 \mathrm{H}) ;{ }^{13} \mathrm{C} \mathrm{NMR}\left(75 \mathrm{MHz}, \mathrm{CDCl}_{3}\right) \delta$ 27.0, 38.7, 62.2, 74.5, 87.3, 128.6, 128.8, 128.9, 129.9, 133.8, 142.2, 164.8, 177.9, 192.3.; FT-IR (neat, $\mathrm{cm}^{-1}$ ) 3433, 2951, 2911, 2833, 1721, 1600, 1438, 1361, 1203, 1003, 882; HRMS (APCITOF) calcd for $\mathrm{C}_{13} \mathrm{H}_{11} \mathrm{NaO}_{4}[\mathrm{M}+\mathrm{Na}]^{+}:$254.0555, found: $254.0553 ;[\alpha]_{\mathrm{D}}^{25}=-82.1\left(\right.$ c $\left.1.25, \mathrm{CHCl}_{3}\right)$.

\subsection{Two-directional reaction (Scheme 5)}

To a solution of $\mathrm{PdCl}_{2}[(S)$-BINAP] $(3.2 \mathrm{mg}, 0.004 \mathrm{mmol})$ in $\mathrm{CH}_{2} \mathrm{Cl}_{2}(1.0 \mathrm{~mL})$ was added $\mathrm{AgSbF}_{6}(3.0 \mathrm{mg}, 0.0088 \mathrm{mmol})$ at room temperature under an argon atmosphere. After stirring for $30 \mathrm{~min}, \mathrm{Et}_{2} \mathrm{O}(1.0 \mathrm{~mL})$, ethyl trifluoropyruvate $2(32 \mu \mathrm{L}, 0.24$ $\mathrm{mmol})$ and $4 \mathbf{a}(32 \mu \mathrm{L}, 0.2 \mathrm{mmol})$ were added to the mixture at $78{ }^{\circ} \mathrm{C}$. The reaction mixture was stirred at $-78{ }^{\circ} \mathrm{C}$ for $8 \mathrm{~h}$, and then freshly distilled ethyl glyoxylate $(61.3 \mathrm{mg}, 0.6 \mathrm{mmol})$ was added at $-78{ }^{\circ} \mathrm{C}$. After stirring for $12 \mathrm{~h}$ at room temperature, the reaction mixture was directly loaded onto a short silica-gel column (hexane/AcOEt $=1 / 1$ ) to remove the catalyst. To a solution of the crude product in THF $(2.0 \mathrm{~mL})$ was added $1 \mathrm{M}$ $\mathrm{HCl}(1.0 \mathrm{~mL})$. After stirring for $1 \mathrm{~h}$ at room temperature, the mixture was extracted with ether. The combined organic layer was washed with brine, dried over $\mathrm{Na}_{2} \mathrm{SO}_{4}$, and evaporated under reduced pressure. Purification by a silica-gel column chromatography (hexane/AcOEt $=2 / 1$ ) gave the corresponding $(R, R)$-diol 16 (91\% yield). The enantiomeric excess was determined by chiral HPLC analysis $(99 \%$ ee $) .{ }^{1} \mathrm{H}$ NMR (300 $\left.\mathrm{MHz}, \mathrm{CDCl}_{3}\right) \delta 1.25(\mathrm{t}, J=7.2 \mathrm{~Hz}, 3 \mathrm{H}), 1.33(\mathrm{t}, J=7.2 \mathrm{~Hz}, 3 \mathrm{H})$, $3.48(\mathrm{~s}, 1 \mathrm{H}), 4.24(\mathrm{q}, J=7.2 \mathrm{~Hz}, 2 \mathrm{H}), 4.37-4.46(\mathrm{~m}, 2 \mathrm{H}), 4.44(\mathrm{~s}$, $1 \mathrm{H}), 5.16(\mathrm{~s}, 1 \mathrm{H}), 6.39(\mathrm{~d}, J=3.3 \mathrm{~Hz}, 1 \mathrm{H}), 6.57(\mathrm{~d}, J=3.3 \mathrm{~Hz}$, $1 \mathrm{H}) ;{ }^{13} \mathrm{C}$ NMR $\left(75 \mathrm{MHz}, \mathrm{CDCl}_{3}\right) \delta 13.8,13.9,62.7,64.6,66.7$, $75.0\left(\mathrm{q}, J_{\mathrm{C}-\mathrm{F}}=32 \mathrm{~Hz}\right), 109.4,111.4,122.1\left(\mathrm{q}, J_{\mathrm{C}-\mathrm{F}}=284 \mathrm{~Hz}\right)$, 146.2, 152.4, 166.9, 171.0; ${ }^{19} \mathrm{~F}$ NMR $\left(282 \mathrm{MHz}, \mathrm{CDCl}_{3}\right) \delta-76.4$; FT-IR (KBr pellet, $\mathrm{cm}^{-1}$ ) 3500, 3055, 2984, 1739, 1423, 1266, 1224, 1017, 896, 742, 704; HRMS (ESI-TOF) calcd for $\mathrm{C}_{13} \mathrm{H}_{15} \mathrm{~F}_{3} \mathrm{NaO}_{7}[\mathrm{M}+\mathrm{Na}]^{+}: 363.0667$, found: $363.0663 ;[\alpha]_{\mathrm{D}}{ }^{25}=-$ 52.3 (c 0.72, $\mathrm{CHCl}_{3}$ ), 99\% ee; HPLC (column, CHIRALPAK AD-3, Hexane $/ 2$-Propanol $=90 / 10$, flow rate $0.6 \mathrm{~mL} / \mathrm{min}, 20{ }^{\circ} \mathrm{C}$, detection UV $210 \mathrm{~nm}$ ) $t_{\mathrm{R}}$ of major isomer $35.2 \mathrm{~min}, t_{\mathrm{R}}$ of minor isomer $41.6 \mathrm{~min}$. The absolute configuration was tentatively assigned by analogy.

\subsection{Sequential catalytic reactions (Scheme 6) \\ 4.8.1. Sequential catalytic reaction with glyoxylate by Au and Pd Catalysts}

To a solution of the neutral Au chloride complex (3.9 mg, 0.005 mmol), which can lead to the corresponding cationic complex 19a, and $\operatorname{AgNTf}_{2}(1.9 \mathrm{mg}, 0.005 \mathrm{mmol})$ in toluene $(1.0 \mathrm{~mL})$ was added diol $17(62.0 \mathrm{mg}, 0.20 \mathrm{mmol})$ at room temperature and the reaction mixture was stirred for $3 \mathrm{~h}$. At the same time, a solution of $\mathrm{PdCl}_{2}[(S)$-BINAP $](8.0 \mathrm{mg}, 0.01 \mathrm{mmol}), \mathrm{AgSbF}_{6}(7.6 \mathrm{mg}$, $0.022 \mathrm{mmol})$, and ethyl glyoxylate $3(61.3 \mathrm{mg}, 0.6 \mathrm{mmol})$ in 
$\mathrm{CH}_{2} \mathrm{Cl}_{2}(1.0 \mathrm{~mL})$ was prepared according to the general procedure. The solution of glyoxylate $\mathbf{3}$ was then transferred to a solution of diol 17 with a syringe at $-20^{\circ} \mathrm{C}$. After stirring for $24 \mathrm{~h}$ at $0{ }^{\circ} \mathrm{C}$, the reaction mixture was loaded directly onto a short silica-gel column (hexane/AcOEt $=1 / 1$ ) to remove the catalyst. To a solution of the crude product in THF $(2.0 \mathrm{~mL})$ was added $1 \mathrm{M} \mathrm{HCl}(1.0 \mathrm{~mL})$. After stirring for $1 \mathrm{~h}$ at room temperature, the mixture was extracted with ether. The organic layer was washed with brine, dried over $\mathrm{Na}_{2} \mathrm{SO}_{4}$, and evaporated under reduced pressure. Purification by silica-gel chromatography (hexane/AcOEt $=5 / 1$ ) gave the corresponding alcohol product $\mathbf{6 f}$ (57\% yield). The enantiomeric excess was determined by chiral HPLC analysis (99\% ee).

\subsubsection{Sequential catalytic reaction with trifluoropyruvate by Au and Pd catalysts}

To a solution of the neutral Au chloride complex (3.9 mg, 0.005 mmol), which can lead to the corresponding cationic complex 19a, and $\mathrm{AgSbF}_{6}(1.7 \mathrm{mg}, 0.005 \mathrm{mmol})$ in $\mathrm{CH}_{2} \mathrm{Cl}_{2}(0.5 \mathrm{~mL})$ was added diol $18(47.6 \mathrm{mg}, 0.2 \mathrm{mmol})$ at room temperature and the reaction mixture was stirred for $1 \mathrm{~h}$. At the same time, a solution of $\mathrm{PdCl}_{2}\left[(S)\right.$-BINAP] $(8.0 \mathrm{mg}, 0.010 \mathrm{mmol}), \mathrm{AgSbF}_{6}(7.6 \mathrm{mg}$, $0.022 \mathrm{mmol})$, and trifluoropyruvate $2(32 \mu \mathrm{L}, 0.24 \mathrm{mmol})$ in $\mathrm{CH}_{2} \mathrm{Cl}_{2}(0.5 \mathrm{~mL})$ was prepared according to the general procedure. Ether $(1.0 \mathrm{~mL})$ and a solution of trifluoropyruvate 2 were added to the solution of diol $\mathbf{1 8}$ via a syringe at $-40{ }^{\circ} \mathrm{C}$. After stirring for $16 \mathrm{~h}$ at $-40{ }^{\circ} \mathrm{C}$, the reaction mixture was loaded directly onto a short silica-gel column (hexane/AcOEt $=1 / 1)$ to remove the catalyst. Purification by a silica-gel chromatography (hexane/AcOEt $=5 / 1)$ gave the corresponding alcohol product $\mathbf{5 f}$ (62\% yield). The enantiomeric excess was determined by chiral HPLC analysis (99\% ee).

\section{Acknowledgments}

This research was supported by Japan Science and Technology Agency (JST) (ACT-C: Creation of Advanced Catalytic Transformation for the Sustainable Manufacturing at Low Energy, Low Environmental Load), JSPS KAKENHI Grant Number 25410036, Mizuho Foundation for the Promotion of Sciences, and The Noguchi Institute. We thank Central Glass Co., Ltd. for the gift of ethyl trifluoropyruvate. We also thank Takasago International Co. for providing $(S)$-BINAP and $(S)$ SEGPHOS derivatives. We are grateful to Prof. T. Imamoto for the gift of QuinoxP*.

\section{References}

1. For reviews, see: a) Mikami, K.; Itoh, Y.; Yamanaka, M. Chem. Rev. 2004, 104, 1; b) Ma, J.-A.; Cahard, D. J. Fluorine Chem. 2007, 128, 975; c) Shibata, N.; Mizuta, S.; Kawai, H. Tetrahedron: Asymmetry 2008, 19, 2633; d) Ma, J.-A.; Cahard, D. Chem. Rev. 2008, 108, PR1; e) Nie, J.; Guo, H.-C.; Cahard, D.; Ma, J.-A. Chem. Rev. 2011, 111, 455.

2. Corbett, J. W.; Ko, S. S.; Rodgers, J. M.; Gearhart, L. A.; Magnus, N. A.; Bacheler, L. T.; Diamond, S.; Jeffrey, S.; Klabe, R. M.; Cordova, B. C.; Garber, S.; Logue, K.; Trainor, G. L.; Anderson, P. S.; Erickson-Viitanen, S. K. J. Med. Chem. 2000, 43, 2019.

3. a) Schäcke, H.; Schottelius, A.; Döcke, W.-D.; Strehlke, P.; Jaroch, S.; Schmees, N.; Rehwinkel, H.; Hennekes, H.; Asadullah, K. Proc. Natl. Acad. Sci. U.S.A. 2004, 101, 227; b) Betageri, R.; Zhang, Y.; Zindell, R. M.; Kuzmich, D.; Kirrane, T. M.; Bentzien, J.; Cardozo, M.; Capolino, A. J.; Fadra, T. N.; Nelson, R. M.; Paw, Z.; Shih, D.-T.; Shih, C.-K.; Zuvela-Jelaska, L.; Nabozny, G.; Thomson, D. S. Bioorg. Med. Chem. Lett. 2005, 15, 4761; c) Lee, T. W.; Proudfoot, J. R.; Thomson, D. S. Bioorg. Med. Chem. Lett. 2006, 16, 654; d) Song, J. J.; Tan, Z.; Xu, J.; Reeves, J. T.; Yee, N. K.; Ramdas, R.; Gallou, F.; Kuzmich, K.; DeLattre, L.; Lee, H.; Feng, X.; Senanayake, C. H. J. Org. Chem. 2007, 72, 292; e) Biggadike, K.; Bledsoe, R. K.; Coe, D. M.; Cooper, T. W. J.; House, D.;
Iannone, M. A.; Macdonald, S. J. F.; Madauss, K. P.; McLay, I. M.; Shipley, T. J.; Taylor, S. J.; Tran, T. B.; Uings, I. J.; Weller, V.; Williams, S. P. Proc. Natl. Acad. Sci. U.S.A. 2009, 106, 18114; f) Sumiyoshi, T.; Tojo, K.; Urabe, D.; Tobe, M. Tetrahedron: Asymmetry 2011, 22, 153.

4. For reviews, see: a) Bandini, M.; Melloni, A.; Umani-Ronchi, A. Angew. Chem. Int. Ed. 2004, 43, 550; b) Bandini, M.; Melloni, A.; UmaniRonchi, A. Synlett 2005, 1199; c) Tsogoeva, S. B. Eur. J. Org. Chem. 2007, 1701; d) Poulsen, T. B.; Jørgensen, K. A. Chem. Rev. 2008, 108, 2903; e) You, S.-L.; Cai, Q.; Zeng, M. Chem. Soc. Rev. 2009, 38, 2190; f) Zeng, M.; You, S.-L. Synlett 2010, 1289; g) Terrasson, V.; de Figueiredo, R. M.; Campagne, J. M. Eur. J. Org. Chem. 2010, 2635; h) Rueping, M.; Nachtsheim, B. J. Beilstein J. Org. Chem. 2010, 6, No. 6.

5. For catalytic asymmetric Friedel-Crafts alkylation using trifluoropyruvate, see: a) Gathergood, N.; Zhuang, W.; Jørgensen, K. A. J. Am. Chem. Soc. 2000, 122, 12517; b) Zhuang, W.; Gathergood, N.; Hazell, R. G.; Jørgensen, K. A. J. Org. Chem. 2001, 66, 1009; c) Corma, A.; García, H.; Moussaif, A.; Sabater, M. J.; Zniber, R.; Redouane, A. Chem. Commun. 2002, 1058; d) Török, B.; Abid, M.; London, G.; Esquibel, J.; Török, M.; Mhadgut, S. C.; Yan, P.; Prakash, G. K. S. Angew. Chem. Int. Ed. 2005, 44, 3086; e) Lyle, M. P. A.; Draper, N. D.; Wilson, P. D. Org. Lett. 2005, 7, 901; f) Zhuang, W.; Poulsen, T. B.; Jørgensen, K. A. Org. Biomol. Chem. 2005, 3, 3284; g) Zhao, J.-L.; Liu, L.; Sui, Y.; Liu, Y.-L.; Wang, D.; Chen, Y.-J. Org. Lett. 2006, 8, 6127; h) Dong, H.-M.; Lu, H.-H.; Lu, L.-Q.; Chen, C.-B.; Xiao, W.-J. Adv. Synth. Catal. 2007, 349, 1597; i) Nakamura, S.; Hyodo, K.; Nakamura, Y.; Shibata, N. Adv. Synth. Catal. 2008, 350, 1443; j) Zhao, J.-L.; Liu, L.; Gu, C.-L.; Wang, D.; Chen, Y.-J. Tetrahedron Lett. 2008, 49, 1476; k) Nie, J.; Zhang, G.-W.; Wang, L.; Zheng, D.-H.; Zheng, Y.; Ma, J.-A. Eur. J. Org. Chem. 2009, 3145; 1) Hui, Y.; Chen, W.; Wang, W.; Jiang, J.; Cai, Y.; Lin, L.; Liu, X.; Feng, X. Adv. Synth. Catal. 2010, 352, 3174; m) Kashikura, W.; Itoh, J.; Mori, K.; Akiyama, T. Chem, Asian J. 2010, 5, 470; n) Wolf, C.; Zhang, P. Adv. Synth. Catal. 2011, 353, 760; o) Doherty, S.; Knight, J. G.; Mehdi-Zodeh, H. Tetrahedron: Asymmetry 2012, 23, 209.

6. Catalytic asymmetric Friedel-Crafts alkylation using fluoral, see: Ishii, A.; Soloshonok, V. A.; Mikami, K. J. Org. Chem. 2000, 65, 1597.

7. Catalytic asymmetric Friedel-Crafts alkylation using trifluoromethyl ketones, see: Blay, G.; Fernández, I.; Monleón, A.; Pedro, J. R.; Vila, C. Org. Lett. 2009, 11, 441.

8. Catalytic asymmetric Friedel-Crafts alkylation using glyoxylate, see: a) Yuan, Y.; Wang, X.; Li, X.; Ding, K. J. Org. Chem. 2004, 69, 146; b) Li, H.; Wang, Y.-Q.; Deng, L. Org. Lett. 2006, 8, 4063; c) Majer, J.; Kwiatkowski, P.; Jurczak, J. Org. Lett. 2009, 11, 4636; For the use of perfluoroalkyl glyoxylate, see: Tonoi, T.; Mikami, K. Eur. J. Org. Chem. 2007, 1730.

9. Aikawa, K.; Hioki, Y.; Mikami, K. Chem. Asian J. 2010, 5, 2346.

10. a) Aikawa, K.; Kainuma, S.; Hatano, M; Mikami, K. Tetrahedron Lett. 2004, 45, 183; b) Mikami, K.; Kawakami, Y.; Akiyama, K.; Aikawa, K. J. Am. Chem. Soc. 2007, 129, 12950; c) Aikawa, K.; Hioki, Y.; Mikami, K. J. Am. Chem. Soc. 2009, 131, 13922; d) Aikawa, K.; Hioki, Y.; Mikami, K. Org. Lett. 2010, 12, 5716; e) Aikawa, K.; Mimura, S.; Numata, Y.; Mikami, K. Eur. J. Org. Chem. 2011, 62; f) Aikawa, K.; Honda, K.; Mimura, S.; Mikami, K. Tetrahedron Lett. 2011, 52, 6682; g) Mikami, K.; Aikawa, K.; Aida, J. Synlett 2011, 2719; h) Aikawa, K.; Hioki, Y.; Shimizu, N.; Mikami, K. J. Am. Chem. Soc. 2011, 133, 20092.

11. QuinoxP*: Imamoto, T.; Sugita, K.; Yoshida, K. J. Am. Chem. Soc. 2005, 127, 11934.

12. See, reference 9; Even in the presence of 0.005 and $0.002 \mathrm{~mol} \% \mathrm{Pd}$ complex, the Friedel-Crafts type reaction with $\mathbf{4 a}$ proceeded to give product $\mathbf{6 a}$ in $75 \%, 98 \%$ ee and $46 \%, 98 \% e e$, respectively (reaction conditions: toluene, $0{ }^{\circ} \mathrm{C}, 72 \mathrm{~h}$ ).

13. a) Harris, J. M.; Karanen, M. D.; O’Doherty, G. A. J. Org. Chem. 1999, 64, 2982; b) Harris, J. M.; O’Doherty, G. A. Tetrahedron Lett. 2000, 41, 183; c) Burke, M. D.; Berger, E. M.; Schreiber, S. L. J. Am. Chem. Soc. 2004, 126, 14095; d) Li, M.; Scott, J.; O’Doherty, G. A. Tetrahedron Lett. 2004, 45, 1005; e) Babu, R. S.; Guppi, S. R.; O'Doherty, G. A. Org. Lett. 2006, 8, 1605; f) Cheng, K.; Kelly, A. R.; Kohn, R. A.; Dweck, J. F.; Walsh, P. J. Org. Lett. 2009, 11, 2703; g) Nicolaou, K. C.; Aversa, R. J.; Jin, J.; Rivas, F. J. Am. Chem. Soc. 2010, 132, 6855; h) Burke, C. P.; Swingle, M. R.; Honkanen, R. E.; Boger, D. L. J. Org. Chem. 2010, 75, 7505; i) Noutsias, D.; Kouridaki, A.; Vassilikogiannakis, G. Org. Lett. 2011, 13, 1166

14. Paolucci, C.; Rosini, G. Tetrahedron: Asymmetry 2007, 18, 2923.

15. a) Achmatowicz, O.; Bukowski, P.; Szechner, B.; Zamojski, A. Tetrahedron 1971, 27, 1973; b) Achmatowicz, O.; Bielski, R. Carbohydr. Res. 1977, 55, 165

16. Egi, M.; Azechi, K.; Akai, S. Org. Lett. 2009, 11, 5002.

17. a) Hashmi, A. S. K.; Sinha, P. Adv. Synth. Catal. 2004, 346, 432; b) Yao, T.; Zhang, X.; Larock, R. C. J. Am. Chem. Soc. 2004, 126, 11164; c) 
Zhang, J.; Schmalz, H.-G. Angew. Chem. Int. Ed. 2006, 45, 6704; d) Schwier, T.; Sromek, A. W.; Yap, D. M. L.; Chernyak, D.; Gevorgyan, V. J. Am. Chem. Soc. 2007, 129, 9868; e) Bew, S. P.; El-Taeb, G. M. M.; Jones, S.; Knight, D. W.; Tan, W.-F. Eur. J. Org. Chem. 2007, 5759; f) Xiao, Y.; Zhang, J. Angew. Chem. Int. Ed. 2008, 47, 1903; g) Kramer, S.; Madsen, J. L. H.; Rottländer, M.; Skrydstrup, T. Org. Lett. 2010, 12, 2758; h) He, C.; Guo, S.; Ke, J.; Hao, J.; Xu, H.; Chen, H.; Lei, A. J. Am. Chem. Soc. 2012, 134, 5766; i) Kramer, S.; Skrydstrup, T. Angew. Chem. Int. Ed. 2012, 51, 4681.

18. a) Ozawa, F.; Kubo, A.; Matsumoto, Y.; Hayashi, T.; Nishioka, E.; Yanagi, K.; Moriguchi, K. Organometallics 1988, 7, 1761; b) Aikawa, K.; Miyazaki, Y.; Mikami, K. Bull. Chem. Soc. Jpn. 2012, 85, 201.

19. Tamsyn, M.; Nikoletta, S.; Manolis, S.; Maria, T.; Georgios, V. Org. Lett. 2005, 7, 2357.

20. Yu, Y. P.; Liepin'sh, E. E.; Bruveris, Z. P.; Lukevits, E. Chem. Hetero. Comp. 1990, 26, 495.

21. Guillard, J.; Lamazzi, C.; Meth-Cohn, O.; Rees, C. W.; White, A. J. P.; Williams, D. J. J. Chem. Soc. Perkin Trans. 2 2001, 1304.

22. Albertin, L.; Bertarelli, C.; Capelli, C. S.; Gallazzi, C. M.; Meille, V. S. J. Chem. Soc. Perkin Trans. 2 2002, 1752.

23. Giancarlo, A.; Colin, E ; John G. S. J. Organomet. Chem. 1981, 204, 153.

24. Elizabeth M. B.; Matthew J. G.; Richard. H. Angew. Chem. Int. Ed. 2008 , $47,3004$.

25. Jeffery, T. Tetrahedron Lett. 1999, 40, 1673.

26. Lee, B. S.; Gil, J. M.; Oh, D. Y. Tetrahedron Lett. 2001, 42, 2345. 


\section{Graphical Abstract}

Catalytic and highly enantioselective Friedel-Crafts

Leave this area blank for abstract info. type reactions of heteroaromatic compounds with trifluoropyruvate and glyoxylate by dicationic palladium complex

Kohsuke Aikawa, Yuya Asai, Yūta Hioki, Koichi Mikami*

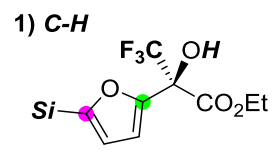

$93 \%, 99 \%$ ee

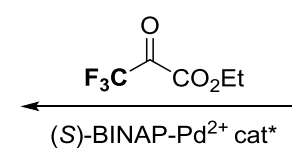

(S)-BINAP-Pd ${ }^{2+}$ cat $^{*}$
$\mathrm{H}^{\mathrm{O}} \mathrm{C}^{\mathrm{S} i}$
2) C-Si

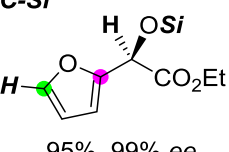

